February 21, 199617:16

\title{
A GENERAL EQUILIBRIUM SPATIAL MODEL OF HOUSING QUALITY AND QUANTITY
}

\author{
by \\ Richard Arnott*, Ralph Braid**, \\ Russell Davidson***, and David Pines****
}

February 1996

Funding for this research from the Canada Mortgage and Housing Corporation and the Foerder Institute for Economic Research, as well as helpful comments from Konrad Stahl and two referees, are gratefully acknowledged.

* Boston College, U.S.A.

** Wayne State University, U.S.A.

*** GREQE, Marseilles, France, and Queen's University, Canada

**** Tel-Aviv University, Israel 
February 21, 199617:16

\section{ABSTRACT AND HEADNOTE}

This paper examines the properties of stationary-state general equilibrium in a monocentric city with durable housing. On the demand side, identical households choose location, housing quality and quantity (floor area), and other goods. On the supply side, developers choose the structural density and time path of quality (which depends on construction quality and maintenance) of buildings. Under a certain set of assumptions, existence and uniqueness of equilibrium are proved, and its comparative static/dynamic properties are determined.

JEL nos: R1, R2

Editorial communications should be sent to:

Prof. Richard Arnott

Department of Economics

Boston College

Chestnut Hill, MA 02167

U.S.A. 
February 21, 199617:16

\section{INTRODUCTION AND OVERVIEW OF THE MODEL}

In the last 30 years, considerable progress has been made in modeling competitive housing markets. Prior to 1980, there were three broad lines of development. First, the monocentric city model of the "new urban economics" (see Alonso (1964)) was extended in many ways, among others to treat non-durable housing, as in Mills (1967) and Muth (1969). Second, durable housing was incorporated into a growing monocentric city (e.g. Fujita (1976) and Anas (1978)end the value and indestructible. Third, Sweeney (1974a, 1974b) and Braid (1979) developed non-spatial, stationary-state filtering models of the housing market in which the quality deterioration of a housing unit depends on the endogenous level of maintenance.

An ultimate objective of these lines of research is to develop a general equilibrium, nonstationary, spatial model of the housing market with the following characteristics. On the demand side, heterogeneous households choose location and other goods, as well as housing quality and quantity. On the supply side, developers choose (with perfect foresight, for each plot of land) construction date, initial quality, and structural density of the first building on the site, the time path of maintenance over the building's life, and the date of demolition (or redevelopment), and so on for subsequent buildings.

Since 1980, a number of authors have constructed spatial models of growing urban areas with durable housing. They all make significant simplifying assumptions. Models with perfect foresight (Arnott (1980), Fujita (1982), Hochman and Pines (1982), Wheaton (1982a), Braid $(1988,1991))$ normally assume that housing cannot be demolished and redeveloped, although Akita and Fujita (1982) allow some limited redevelopment. Models with demolition and redevelopment (Brueckner (1980a, 1980b), Wheaton (1982b), Cooke and Hamilton (1984)) normally assume myopic expectations. Brueckner (1981) assumes a stationary-state city, and incorporates both perfect foresight and multiple rounds of redevelopment on each plot of land. All these papers ignore consumer choice over more than a single housing attribute, ignore endogenous 
February 21, 199617:16

maintenance expenditures (except that Brueckner (1981) allows endogenous maintenance expenditures that are constrained to be constant over time), and assume a single income group (except Brueckner (1980b), Cooke and Hamilton (1984), and Braid (1991)).

In this paper, each developer chooses construction date, initial quality, structural density, the time path of maintenance, and the date of demolition and reconstruction for a succession of buildings on his/her site. However, the economic environment and the rent gradient (giving rent as a function of quality and location) remain constant over time, and as a result we may say that the equilibrium is a stationary state. We consider only the case with a single income group, and some additional restrictive assumptions are employed. Nevertheless, this paper makes a number of advances over the previous literature. It is the first paper to treat household choice over both quality and quantity. ${ }^{1}$ More significantly, by continuing the line of research of Arnott, Davidson, and Pines (1983), referred to hereafter as ADP1, and Arnott, Davidson, and Pines (1986), referred to hereafter as ADP2, it provides the first general equilibrium spatial model of the housing market to treat quality and endogenous maintenance. It also provides the first proof of existence and uniqueness in a monocentric city with internal ownership of land?

It is worthwhile to place our model in the context of the Arrow-Debreu model of perfect competition. Temporarily suppose, to be consistent with Debreu (1959), that time, location, structural density, and quality are all discrete. Then housing is a set of commodities indexed by time, location, structural density, and quality. Each of these commodities can be produced using a

\footnotetext{
${ }^{1}$ One on-going debate in the housing literature concerns how to treat the multi-dimensional heterogeneity of housing units. In the classical, Muthian model (1969) all housing characteristics are aggregated into a single composite index, termed the "quantity of housing services." At the other extreme, in the hedonic price literature, demand is expressed in terms of all housing characteristics. The problem with the Muthian approach is that it is too coarse for many purposes; policy makers are often interested in the effects of policy on housing quality and the volume and nature of housing construction, information the Muthian approach cannot provide. There are two main problems with the hedonic price approach. First, it is too complex. Demand and technological parameters are hard to estimate, particularly in view of the insufficient detail of most of the housing data that has been collected. Second, housing units are sparse in characteristics space; thus, it may be seriously misleading to view the household as able to buy any bundle of housing characteristics it wishes. The treatment of housing in this paper lies between these two extremes.

${ }^{2}$ See Fujita and Smith (1987) for a proof of existence and uniqueness of equilibrium in a monocentric city model without housing or internal ownership of land, but with multiple income groups. See also the references therein.
} 
February 21, 199617:16

variety of Leontief techniques. ${ }^{3}$ This multiplicity of techniques can render the production set nonconvex. On the consumer side, in each time period a household may consume housing of only one quality and at a single location, which implies nonconvexity of the consumption set. It is these structural nonconvexities that distinguish the model from the Arrow-Debreu model of perfect competition, and which cause the proof of existence of equilibrium to be non-trivial.

The paper incorporates an extended version of a partial equilibrium developer model introduced in ADP1 and ADP2, an extended version of a household decision model used in ADP2, and a market-clearing spatial model. The extensions include the simultaneous treatment of structural density, quality, and quantity of housing consumption. The treatment of structural density on the producer side is absent in ADP1 (though present in ADP2), and the treatment of quantity on the consumer side is absent in both. The market-clearing spatial model connects the two extended submodels of households and producers and allows comparative statics in the general equilibrium context.

A building is constructed at a particular structural density and initial quality. As it ages, its structural density remains the same, but its quality can change, and so too can the floor area of the housing units it contains. Quality deteriorates continuously through time at a rate which depends on maintenance expenditure per unit floor area, and can be negative. The floor area of the housing units can be altered without cost. These assumptions ensure that, in competitive equilibrium, there is a well-defined rent per unit floor area for housing as a function of quality and location.

A household derives utility from the quality and floor area of its housing, as well as from other goods. It is indifferent to structural density and to location except through the effect of the

\footnotetext{
${ }^{3}$ For example, where $t$ is time, $x$ location, $q$ quality, $\mu$ structural density, $Q$ quantity of housing, $C$ quantity of composite good (either maintenance or construction),

$$
Q_{t, x, q, \mu}=\min \left(Q_{t-1, x, q+1, \mu}, \frac{C_{t}}{\beta_{q+1}}\right)
$$

specifies that housing of type $t, x, q, \mu$ can be produced using housing of the same structural density and location from a period earlier of the next higher quality, when combined with the appropriate amount of composite good (maintenance expenditures) from period $t$, where $\beta_{q+1}$ is an exogenous parameter.
} 
February 21, 199617:16

latter on transportation costs. When purchasing housing services of a particular quality and at a particular location, the household faces a market-determined price and can choose how many units of floor area to rent at that price.

We consider a stationary state equilibrium in the sense that the market price of a unit of floor area of a given quality at a given location does not vary with time.

The housing market studied in this paper is that of a monocentric urban area. Each household spends on transportation a sum which depends on the distance, denoted $x$, of its residential location from the city center (where all the non-residential activities take place).

The interrelationships of the model are described in chart 1. There are three submodels. The first is a demand-side model. From an exogenous utility function for the identical households are derived a bid-rent function $P(q, x)$-- the demand price for one unit of housing of quality $q$ at location $x$-- and a housing demand function $H(q, x)$-- the quantity demanded of housing of quality $q$ at $x$ when prices are given by $P(q, x)$. Exogenous to this submodel are household income, $Y$, the household utility level, $U$, and the level of transport costs, denoted by a shift parameter $\alpha$. Next is a supply-side model, an extension of the spatial intertemporal model presented in ADP2. This model takes the rent function $P(q, x)$ at some location $x$ as exogenous and independent of time, and generates the optimal program for a landlord-builder, called a developer, at $x$, giving the (structural) density $\mu$ of housing built there, the quality $q_{0}$ of initial construction, the program of maintenance over the life of the building, $m(t)$, and the timing of demolitions and reconstructions if these occur. The discounted value, $\Pi$, of the profit stream to the developer, which can be interpreted as the competitive land value, is also calculated. ${ }^{4}$ Exogenous to this model are the construction and maintenance technologies, characterized by functions $K\left(q_{0}, \mu\right)$ and $g(q, m)$ to be defined later, and the "agricultural rent," $R_{A}$, the opportunity cost of undeveloped land. Finally comes the equilibrium or market-clearing model, in which two

\footnotetext{
${ }^{4}$ Under competition, these profits would be capitalized into land values. Thus, $\Pi$ may be interpreted to be land value in excess of the land's value in agricultural use (or differential land value).
} 
February 21, 199617:16

more exogenous variables appear, the population, $\bar{N}$, of the (closed) city, and the non-rental income, $I$, of the (identical) households. The first two submodels are put together and the following variables are determined: the equilibrium rent function over quality and location, total household income, $Y$ (consisting of $I$ plus an equal share of development profits), utility $U$, the pattern over the city of housing density, quality levels, demolition rates and so forth, as well as the total city area.

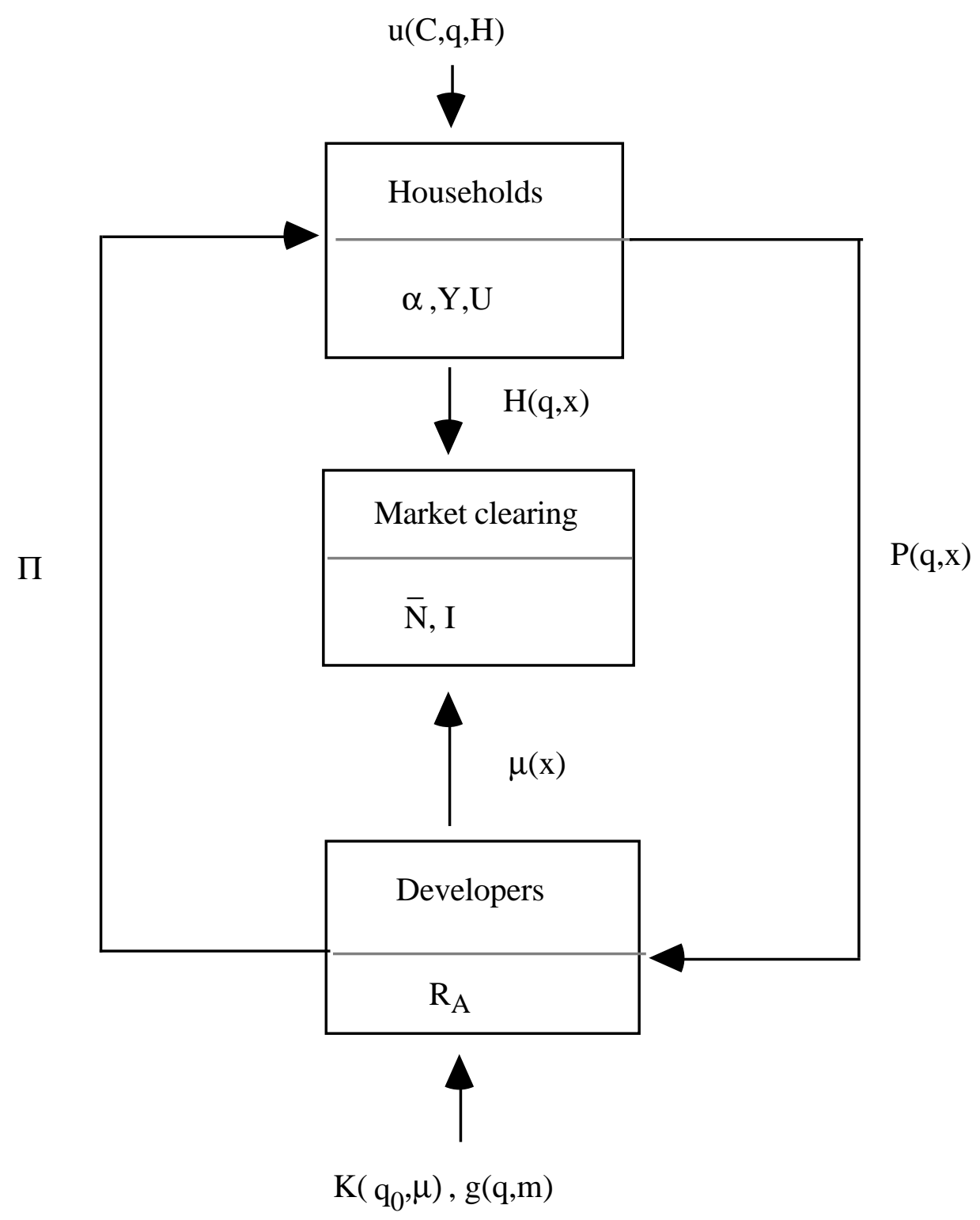

Chart 1: Relationships between the submodels 
February 21, 199617:16

Section 2 describes the household model and the derivation of $P(q, x)$ and $H(q, x)$. It includes comparative statics regarding the effects of $\alpha, U$, and $Y$ on these functions. Section 3 is devoted to the dynamic developer problem and it includes comparative statics regarding the effects of $P(q, x), K\left(q_{0}, \mu\right)$, and $R_{A}$. Section 4 presents the market-clearing model, and examines issues of existence and uniqueness. Section 5 examines its comparative static properties, investigating the effects of changes in $I, \bar{N}, \alpha, R_{A}$, and $K\left(q_{0}, \mu\right)$ on the structure of the city. ${ }^{5}$ Concluding comments are presented in Section 6.

\section{DEMAND-SIDE MODEL}

The utility of a representative household is given by

$$
U=u(C, H, q)
$$

where $C$ is the quantity consumed of a Hicksian composite of non-housing goods; $H$ is housing quantity; $q$ is housing quality; and $u(\cdot)$ is differentiable strictly, quasiconcave for each $q$, with $\partial u / \partial C>0, \partial u / \partial H>0$, and $\partial u / \partial q>0$. Quantity, $H$, as floor area, is a cardinal measure. Quality, on the other hand, is an ordinal measure, such that $q=0$ denotes the lowest possible quality.

It is convenient to characterize tastes by the expenditure function dual to the utility function (1):

$$
E(U, P, q)=\min _{C, H}(C+P H \mid u(C, H, q) \geq U)
$$

$P$ is here interpreted as the price (or rent) of one unit of floor area of quality $q$. If a household lives in housing of quality $q$, pays $P$ for each unit of floor space, and receives a utility level of $U$,

${ }^{5}$ Section 5 derives the comparative static results for $I$. The derivation of the other comparative static results is presented in an unpublished appendix which is available from the authors on request. 
February 21, 199617:16

then by Shephard's Lemma the quantity of housing it will choose to consume is

$$
h(U, P, q) \equiv E_{P}(U, P, q)
$$

the partial derivative of $E$ with respect to its argument $P$. Consequently, the amount of the composite good consumed will be

$$
c(U, P, q) \equiv E(U, P, q)-P h(U, P, q)
$$

We assume that households can neither borrow nor save. Letting $Z$ denote household income net of transportation costs, we can then define the bid rent (see Wheaton (1977)) on housing of quality $q$, conditional on $U$ and $Z$. This bid rent is the maximum rent per unit floor area of quality $q$ consistent with the attaining of a utility level $U$ by a utility-maximizing household with net income $Z$. If the bid-rent function is expressed as $P(q, U, Z)$, then it is defined by the identity

$$
E(U, P(q, U, Z), q) \equiv Z
$$

Differentiating (3) with respect to $q, U$ and $Z$, and using Shephard's Lemma yields

$$
\begin{aligned}
& P_{q}=-E_{q} / E_{P}=-E_{q} / h(U, P, q)>0, \\
& P_{U}=-E_{U} / E_{P}=-E_{U} / h(U, P, q)<0, \\
& P_{Z}=1 / E_{P}=1 / h(U, P, q)>0 .
\end{aligned}
$$

In order to establish subsequently the existence of a non-trivial equilibrium on the housing market, we must impose some restrictions on preferences. First, we require that utility be defined on the entire (open) orthant, which would not be the case if, for example, the underlying preferences entailed a survival bundle or commitment expenditures (e.g. a Stone-Geary utility function). Second, we require that, for any given level of utility $U$, quality $q$, and rent $P$, both the quantity of housing and the quantity of other goods consumed are strictly positive. Further, as $P$ 
February 21, 199617:16

tends to infinity, we require that expenditure on housing also tend to infinity. Formally, we assume:

(AS-1a) (i) For every $Z>0, q>0$, and $P>0$, there is a solution $U(Z, P, q)$ to the equation $E(U, P, q)=Z$.

(ii) For every $q>0, P>0$, and $U, E_{P}(U, P, q)=h(U, P, q)>0$ and $c(U, P, q)>0$.

(iii) For every $q>0$ and $U, \lim _{P \uparrow_{\infty}} P h(U, P, q)=\infty$.

We make the following additional assumptions on preferences: the composite good is non-inferior; housing quantity is strictly normal; and housing quality is normal in the sense that the marginal willingness to pay for quality, $P_{q}(q, U, Z)$, rises with utility. Thus,

$$
c_{U}(U, P, q) \geq 0 \quad h_{U}(U, P, q)>0 \quad P_{q U}(q, U, Z)>0 .
$$

For net income $Z$ we take the following specification:

$$
Z=Y-\alpha s(x)
$$

where: $x$ is distance from the city center; $s(x)$ is the transport cost function, with $s$ continuous, $s^{\prime}$ positive and finite, $s(0)=0$, and $0<s(x)<\infty$ for $0<x<\infty ; \alpha$ is a shift parameter; and $Y$ is gross household income. Plainly $Z_{Y}>0, Z_{\alpha}<0$ for $x>0, Z_{x}<0$.

It is convenient to define an equilibrium housing quantity demand function by the relation

$$
H(q, U, Z) \equiv h(U, P(q, U, Z), q)=E_{P}(U, P(q, U, Z), q)
$$

Total differentiation of (6) under (AS-lb) gives, by use of (4),

$$
H_{U}=h_{P} P_{U}+h_{U}>0, \quad H_{Z}=h_{P} P_{Z}<0,
$$


February 21, 199617:16

since $h_{P}=E_{P P}<0$ by the concavity of the expenditure function. Furthermore,

$$
H_{q}=h_{P} P_{q}+h_{q} \gtreqless 0
$$

is of indeterminate sign since $h_{P}<0, P_{q}>0$, and $h_{q}$ is of indeterminate sign. Also, from (4) and

$$
H(q, U, Z) P_{Z}(q, U, Z)=1 .
$$

Total differentiation of this result gives:

$$
P_{Z Z}=-\frac{H_{Z}}{H^{2}}>0 \quad P_{Z U}=-\frac{H_{U}}{H^{2}}<0 \quad P_{Z q}=-\frac{H_{q}}{H^{2}},
$$

this last cross partial derivative being of indeterminate sign. Note that the use of (5) allows us to obtain all the partial derivatives with respect to the variables $Y, \alpha$, and $x$ that we shall need subsequently.

The following assumption plays a key role in much of our analysis:

$$
H_{q}=0 .
$$

The assumption states that, for fixed household net income and utility, the quantity of housing demanded is independent of the quality of that housing. 6,7 An important consequence follows from (9), namely that

$$
P_{q Z}=0
$$

\footnotetext{
${ }^{6} \mathrm{An}$ expression for $H_{q}$ in terms of the direct utility function may be obtained from total differentiation of $u(Z-p H, H, q)=U$ and $-u_{C} p+u_{H}=0$. The resulting expression implies that $H_{q}=0$ is equivalent to $H \frac{u_{H}}{u_{C}}\left(-u_{C C} u_{q}+u_{C} u_{C q}\right)+u_{q} u_{C}-u_{C} u_{H q} H=0$. A class of utility functions for which $H_{q}=0$ is $u(C, H, q)=\min (v(C, q), H)$.

${ }^{7} \mathrm{~A}$ point of disagreement in the literature on urban spatial models with durable housing is whether it is more reasonable to assume that, over the lifetime of a building, apartment size is fixed or costlessly adjustable. Under (AS-2) this point of disagreement is immaterial. As the building deteriorates, the size of apartment most preferred by households remains fixed. Consequently, the developer has no incentive to adjust apartment size as the building ages.
} 
February 21, 199617:16

This implies that the slope of the bid-rent function, $P_{q}$, is independent of $Z$ and therefore of income and location, for $U$ fixed. We use (AS-2) in our proof of existence and also to sign a number of comparative statics derivatives that are otherwise ambiguous.

In Table 1, we bring together those results of this section on the signs of partial derivatives that will be used subsequently.

\section{3. $\quad$ SUPPLY-SIDE MODEL}

This submodel has been treated in detail in ADP2. The developer of a particular piece of land at location $x$ determines, as a consequence of the rent function $P(q)$ there, whether to construct housing, whether eventually to demolish it and reconstruct, and the values of the following other variables characterizing the building: $q_{0}$, quality at time of construction; $q_{T}$, quality at time of demolition; $T$, age at time of demolition; $m(\tau)$, flow of maintenance expenditure at age $\tau$; and $\mu$, density, i.e. number of quantity units built per unit area of land.

The developer maximizes profit per unit of land area, defined as

$$
\Pi\left(q_{0}, q_{T}, T, \mu\right)=\frac{\mu J\left(q_{0}, q_{T}, T\right)-K\left(q_{0}, \mu\right)}{1-e^{-r T}}-\frac{R_{A}}{r},
$$

where: $r$ is the discount rate; $R_{A}=$ agricultural rent (per unit time); $K\left(q_{0}, \mu\right)$ is the cost per unit area of land of constructing a building of quality $q_{0}$ and density $\mu$; and $J\left(q_{0}, q_{T}, T\right)$ is the solution to the following maximization problem over $m(\tau)$ :

$$
J\left(q_{0}, q_{T}, T\right)=\max _{m(\tau)} \int_{0}^{T} e^{-r \tau}(P(q)-m) d \tau
$$

subject to $q(0)=q_{0}, q(T)=q_{T}$, and the maintenance technology linking $m$ and $q$, namely

$$
\dot{q}=g(q, m)
$$


February 21, 199617:16

The assumptions made for regularity of the solution to this model are (see ADP2 for discussion and more details):

(AS-4) There exists a positive $\hat{q}$ such that for all $q \in(0, \hat{q})$, there is an $m>0$ for which $g(q, m)=0$. Also, this $m$ tends to zero as $q \rightarrow 0$.

$$
\lim _{m \downarrow 0} g_{m}(q, m)=\infty, \quad \lim _{m \uparrow \infty} g_{m}(q, m)=0, \quad g_{m m}<0, \text { for all } q>0 .
$$
$K(0, \mu)=K(q, 0)=0 ; K_{q}, K_{\mu}, K_{\mu \mu}, K_{q \mu \mu}>0$ for $q \in(0, \hat{q})$ and $\mu>0.8$

The next two assumptions, which concern the interaction of the utility function $u$ (and hence $P$ ) and the technology functions $K$ and $g$, require for their formulation the use of the phase plane for problem (12). This is depicted in Figure 1, in which $\phi$ denotes the co-state variable of the problem, the shadow price of quality. The current-valued Hamiltonian for (12) is found by maximizing $P(q)-m+\phi g(q, m)$ with respect to $m$ :

$$
\mathrm{H}(q, \phi)=\max _{m \geq 0}(P(q)-m+\phi g(q, m))
$$

and the locus marked $\dot{q}=0$ in Figure 1 is the set of points $(q, \phi)$ satisfying $\mathrm{H}_{\phi}(q, \phi)=0$. The curve CDGF is the optimal path for the particular configuration of the phase plane drawn, and it is a solution of the canonical equations

$$
\begin{aligned}
& \dot{q}=\mathrm{H}_{\phi}(q, \phi) \\
& \dot{\phi}=r \phi-\mathrm{H}_{q}(q, \phi)=r \phi-P_{q}-\phi g_{q} .
\end{aligned}
$$

The locus marked $\phi=K_{q} / \mu$ is the locus of points satisfying the first-order condition for $q_{0}$ in the maximization of (11) for a particular value of $\mu$, and is defined by the equation $\phi=K_{q}(q, \mu) / \mu$.

We make the following assumptions about the configuration of the phase plane:

\footnotetext{
${ }^{8}$ Note that all the properties are independent of the cardinalization of $q$, except that $q=0$ is defined to be the lowest possible quality.
} 
February 21, 199617:16

(AS-6) There exists $q_{S} \in(0, \hat{q})$ such that for points on the $\dot{q}=0$ locus, $\dot{\phi} \lesseqgtr 0$ for $q \lesseqgtr q_{S}$ ( $\dot{\phi}$ given by (14)).

(AS-7) There is an upper limit $\bar{\mu}$ such that, for $0<\mu<\bar{\mu}$, the locus $\phi=K_{q} / \mu$ lies entirely below the locus $\dot{q}=0$ for $0<q<\hat{q}$; and there exists a critical value $\tilde{q}$ such that for $q>\tilde{q}$ the slope of the locus is algebraically greater than that of the solution of the canonical equations (13) and (14) through the locus at $q$, and for $q$ $<\tilde{q}$ smaller.

As shown in ADP2, (AS-3) through (AS-7) guarantee the existence of a unique optimal program, specified by $\mu, q_{0}, q_{T}, T, m(\tau)$, a solution to (13) and (14), and maximized profit $\Pi$. If Area A in Figure 1 exceeds Area B, then $T$ is finite, and a demolition cycle path such as CDGF ( with Area $\mathrm{CDG}=$ Area OHGF -- see discussion below) is followed. Otherwise, $T$ is infinite, so that the building once constructed is never demolished, and the stable arm, WS in Figure 1, is followed.

The remainder of this section derives results which are used in the comparative static analysis of Section 5 .

To obtain the comparative static properties of the submodel, we follow the general procedure outlined in the Appendix of ADP2: Let $X$ denote the vector of endogenous variables $\left(q_{0}, q_{T}, T\right)$, and let $\Pi_{X}$ and $\Pi_{X X}$ denote the gradient and Hessian respectively of $\Pi$, as defined by (11), with respect to $X$. If $\lambda$ denotes any shift parameter, then

$$
\frac{d X}{d \lambda}=\frac{\partial X}{\partial \lambda}+\frac{\partial X}{\partial \mu} \frac{d \mu}{d \lambda}
$$

where $\partial / \partial \lambda$ denotes changes in which $\mu$ is held constant, $\partial / \partial \mu$ changes in which $\lambda$ is held constant, and $d / d \lambda$ changes in which $\mu$ adjusts optimally along with $X$. We have, in obvious notation: 
February 21, 199617:16

$$
\begin{aligned}
& \left.\frac{\partial X}{\partial \lambda}=-\Pi_{X X}^{-1} \Pi_{X \lambda}, \quad \frac{\partial X}{\partial \mu}=-\Pi_{X X}^{-1} \Pi_{X \mu} \text { (total differentiation of } \Pi_{X}=0\right) \\
& \left.\frac{d \mu}{d \lambda}=-\frac{\Pi_{\mu \lambda}+\Pi_{\mu X} \partial X / \partial \lambda}{\Pi_{\mu \mu}+\Pi_{\mu X} \partial X / \partial \mu} \text { (total differentiation of } \Pi_{\mu}=0\right) \\
& \left.\Pi_{\mu \mu}+\Pi_{\mu X} \partial X / \partial \mu<0 \text { (second-order condition for optimal } \mu\right) \\
& \frac{d \Pi}{d \lambda}=\Pi_{\lambda} \text { (envelope theorem). }
\end{aligned}
$$

Further, of the three components of $\partial X / \partial \mu$ : If $T$ is finite, the components of $\partial X / \partial \mu$ are as follows: $\partial q_{T} / \partial \mu<0$ but $\partial q_{0} / \partial \mu$ and $\partial T / \partial \mu$ cannot in general be signed; while if $T$ is infinite, $\partial q_{0} / \partial \mu<0$ and $\partial q_{T} / \partial \mu=0$.

We consider four comparative statics exercises. The first is taken from ADP2, and treats a parallel upward shift of the rent function $P(q)$. If we call the shift parameter $\chi$ in this case, then

$$
P(q, \chi)=P(q)+\chi
$$

and $P_{\chi}=1$ for all $q$. Changes in $\chi$ do not affect the canonical equations (13) and (14), and so we see from (12) that $J_{\chi}\left(q_{0}, q_{T}, T\right)=\int_{0}^{T} e^{-r \tau} d \tau=\frac{1}{r}\left(1-e^{-r T}\right)$. Thus, from (11), $\Pi_{\chi}=\mu / r ; \Pi_{\mu \chi}=1 / r$; and $\Pi_{X \chi}=0$. From (16), $\partial X / \partial \chi=0$; from (17) and (18),

$$
\frac{d \mu}{d \chi}>0
$$

from (19),

$$
\frac{d \Pi}{d \chi}=\frac{\mu}{r}
$$

and from (15), $d X / d \chi$ has the same signs as $\partial X / \partial \mu$.

The next exercise deals with a change in the slope of the function $P(q)$. The shift parameter is called $\gamma$, and we put 
February 21, 199617:16

$$
P(q, \gamma)=P(q)+\gamma \ell(q)
$$

where $\ell^{\prime}(q)>0$, and if $q^{*}(\tau)$ denotes the optimal path of quality for $\gamma=0$, then

$$
\int_{0}^{T} e^{-r \tau} \ell\left(q^{*}(\tau)\right) d \tau=0
$$

Condition (22) implies that $\partial J / \partial \gamma=0$. It also implies that $\ell\left(q_{0}\right)>0$ and $\ell\left(q_{T}\right)<0$. From (11), we obtain

$$
\Pi_{\gamma}=0, \quad \Pi_{\mu \gamma}=0
$$

Instead of using (16) to find $\partial X / \partial \gamma$, we have the following direct argument. If $T$ if finite, it is known from ADP1 and ADP2 that the first-order condition for $T$ in the maximization of $\Pi$ implies that the areas CDG and OHGF in Figure 1 must be equal for CDGF to be the optimal path. The locus $\phi=K_{q} / \mu$ is unchanged by shifts in $\gamma$ (for given $\mu$ ) and so is the canonical equation (13), and hence the locus $\dot{q}=0$. However, for (14) we have $\partial \dot{\phi} / \partial \gamma=-P_{q \gamma}=$ $-\ell^{\prime}(q)<0$. Thus, the algebraic slope $d \phi / d q$ of any solution to (13) and (14) with $\dot{q}<0$ increases with $\gamma$. Now consider Figure 2, where EF denotes the optimal path for $\gamma=0$. In order to satisfy the equal-area condition, the optimal path, $\mathrm{CD}$, for some $\gamma>0$, must cut $\mathrm{EF}$, and must do so in the sense shown if its slope is to be greater than that of EF at their intersection. We conclude that $\partial q_{0} / \partial \gamma>0$ and $\partial q_{T} / \partial \gamma>0 . \partial T / \partial \gamma$ cannot be signed in general.9 To obtain $d \mu / d \gamma$ from (17) we need the vector $\Pi_{\mu X}$. It is shown in ADP2 that $\Pi_{\mu q_{0}}<0, \Pi_{\mu q_{T}}=0$, and $\Pi_{\mu T}>0$. From (17), (18), and (23) we see that $d \mu / d \gamma$ has the same sign as $\Pi_{\mu X} \partial X / \partial \gamma$, which, since $\Pi_{\mu q_{T}}=0$ is the sum of $\Pi_{\mu q_{0}} \partial q_{0} / \partial \gamma<0$ and $\Pi_{\mu T} \partial T / \partial \gamma$, which is of indeterminate sign. One may presume that usually $d \mu / d \gamma<0$, but this is not always true. If it is, then from (15) $d q_{T} / d \gamma>0$. If further $\partial q_{0} / \partial \mu<0$ (the most likely case according to ADP2), then $d q_{0} / d \gamma>0$.

\footnotetext{
${ }^{9}$ All we can say is that if $\partial T / \partial \gamma>0$, the profit per construction-demolition cycle, viz. $\mu J-K$, increases with $\gamma$, and if $\partial T / \partial \gamma<0$ it decreases. This follows since $\Pi_{\gamma}=0$ and hence $d \Pi / d \gamma=0$ by (18); since overall profits remain unchanged, profit per cycle must be larger, the longer the cycle.
} 
February 21, 199617:16

The case of $T=\infty$ is now considered. In Figure 3, GS is the optimal path for $\gamma=0$ and HS' the optimal path for $\gamma>0$. Since $\partial \dot{\phi} / \partial \gamma<0$, then an increase in $\gamma$ causes the $\dot{\phi}=0$ curve to shift upwards, and the saddlepoint to move right along the $\dot{q}=0$ line. Furthermore, since with $\mathrm{S}^{\prime}$ to the right of $\mathrm{S}$, an intersection of GS and $\mathrm{HS}^{\prime}$ would violate the result that the algebraic slopes of paths increase with $\gamma$, HS ${ }^{\prime}$ must lie to the right of GS. Thus $\partial q_{0} / \partial \gamma>0$, and $\partial q_{T} / \partial \gamma>0$. Since $\Pi_{\mu q_{0}}<0, \Pi_{\mu q_{T}}=0$, and $\Pi_{\mu T}=0$ (see ADP2), then from (17), (18), and (23), $d \mu / d \gamma<0$. And because $\partial q_{0} / \partial \mu<0$ and $\partial q_{T} / \partial \mu=0$, it follows from (15) that $d q_{0} / d \gamma>0$ and $d q_{T} / d \gamma>0$.

Note that combinations of the previous two comparative statics exercises allows us to treat movements of the rent gradient that can be decomposed into a rotation (in the sense above) and a parallel shift. This will prove useful when we do the general equilibrium comparative statics.

The third comparative statics exercise treats a shift in the construction technology function $K$. The shift parameter is $\delta$ and we put

$$
K\left(q_{0}, \mu ; \delta\right)=\delta K\left(q_{0}, \mu / \delta\right)
$$

It can be seen that if $\delta$ is doubled, then the new cost of constructing a building of a given quality and structural density equals twice the old cost of a building of the same quality but half the density. Since there are increasing marginal construction costs to density, an increase in $\delta$ corresponds to a particular form of technological improvement in construction. This shift has the following properties:

$$
\begin{aligned}
& K\left(q_{0}, \mu ; 1\right)=K\left(q_{0}, \mu\right) \\
& K_{\delta}\left(q_{0}, \mu ; 1\right)=K\left(q_{0}, \mu\right)-\mu K_{\mu}\left(q_{0}, \mu\right)<0(\text { by }(\mathrm{AS}-5)) \\
& K\left(q_{0}, \mu ; 1\right) / \mu=K\left(q_{0}, \mu \delta ; \delta\right) / \mu \delta \\
& K_{q}\left(q_{0}, \mu ; 1\right) / \mu=K_{q}\left(q_{0}, \mu \delta ; \delta\right) / \mu \delta
\end{aligned}
$$


February 21, 199617:16

$$
K_{\mu}\left(q_{0}, \mu ; 1\right)=K_{\mu}\left(q_{0}, \mu \delta ; \delta\right)
$$

Thus changes in $\mu$ and $\delta$ that leave $\mu / \delta$ unchanged leave the $\phi=K_{q} / \mu$ locus unchanged. Since the canonical equations (13) and (14) are unaffected by $\delta$, the whole phase plane is unchanged, and the optimal path for all such $(\mu, \delta)$ pairs is the same. From (11), $\Pi_{\mu}=\frac{1}{1-e^{r T}}\left(J-K_{\mu}\right)$, and this too is unchanged if $\mu / \delta$ is. Thus, if $\mu^{*}$ is optimal for $\delta=1, \delta \mu^{*}$ is optimal for other values of $\delta$. Hence, $d \mu / d \delta=\mu^{*}=\mu / \delta ; d X / d \delta=0$; and

$$
\frac{d \Pi}{d \delta}=\Pi_{\delta}=-\left(1-e^{-r T}\right)^{-1} K_{\delta}>0 .
$$

Finally, consider changes in the agricultural rent $R_{A}$. A change in $R_{A}$ can affect whether a building will be constructed or not, since $\Pi_{R_{A}}=-\frac{1}{r}<0$, and construction requires $\Pi \geq 0$. But otherwise $R_{A}$ is just a fixed cost, and affects none of $q_{0}, q_{T}, T, \mu$.

In Table 2, we bring together those results of this section on the signs of partial derivatives that will be used subsequently.

\section{MARKET-CLEARING MODEL AND EXISTENCE AND UNIQUENESS OF EQUILIBRIUM}

The market-clearing model to be constructed employs the demand-side submodel of section 2 and the supply-side submodel of section 3. Since an essential feature of the supply-side model is that the rent function $P$ does not change over time, conditions must be imposed on the market-clearing model to ensure that $P$ is indeed time-invariant. ${ }^{10}$ It is easy to require that the exogenous functions -- $u, K, g$, and $s$-- be constant, as well as all the exogenous parameters --

\footnotetext{
${ }^{10} \mathrm{We}$ do not investigate in this paper the possibility of an equilibrium with non-constant $P$. This appears to be a difficult matter, and its study would necessitate a quite different, and explicitly time-dependent, supplyside model, complete with a mechanism to generate expectations for future rent profiles. Thus, later assertions of uniqueness of equilibrium in this paper mean (or at least are proved to mean) only uniqueness in the class of equilibria with time-independent rent functions.
} 
February 21, 199617:16

$\alpha, \delta, R_{A}, \bar{N}$ (city population), and $I$ (non-rental income of a household). But $P$ depends also on the variables $Y$ (gross household income) and $U$ (see (3) and (5)), and so we must be sure that they too are constant.

To examine the conditions under which $Y$ and $U$ are in fact constant, we must consider the market-clearing condition itself. For some given time-independent rent function $P(q, x)$, we can derive from the supply-side submodel the optimal density and demolition age as functions of location; we obtain $\mu(x)$ and $T(x)$. If $\theta(x) d x$ denotes the area of land available for development in the ring contained between $x$ and $x+d x$, then the quantity of housing supplied in this ring is $\theta(x) \mu(x) d x$. Let $F(\tau, x, t)$ denote the distribution of building ages in the ring, the proportion of buildings at $x$ of ages $\leq \tau$ at time $t$. Clearly, $F(T(x), x, t)=1$. Next let $q(\tau, x)$ be the optimal quality at $x$ of a building of age $\tau$, calculated again from the supply-side submodel. Now consider the demand side, and let $H(q, x)$ be the quantity of housing demanded by a household at $x$ if the quality is $q$ with rent function $P(q, x)$. Then in market equilibrium the number of households in the ring at $x$ at time $t$ is

$$
\theta(x) \mu(x) d x \int_{0}^{T(x)} \frac{1}{H(q(\tau, x), x)} d F(\tau, x, t)
$$

This expression is, in general, dependent on $t$, as is, by integration over $x$, the total number of households in the city, contrary to the assumption that it is fixed and equal to $\bar{N}$.

There are three situations, however, in which (25) is time invariant with a time independent rent function. In the first, there is a rectangular distribution of building ages, which ensures that $F(\tau, x, t)$ is independent of time; specifically,

$$
F(\tau, x, t)=\tau / T(x) \quad(\tau \leq T(x)) .
$$

In the second, the stable-arm path is profit-maximizing and all housing has reached the corresponding saddlepoint quality, $q^{s}(x)$; then $(25)$ reduces to $\left(\theta(x) \mu(x) / H\left(q^{s}(x), x\right)\right) d x$ 
February 21, 199617:16

independently of $t$. In the third, (AS-2) applies, so that $H_{q}=0$, which implies that $H(q(\tau, x), x)=H(x)$. Then (25) becomes $\frac{\theta(x) \mu(x)}{H(x)} d x$.

The first situation, a rectangular distribution of building ages, appears reasonable at first glance. However, at any location, all developers will have faced the same prices through history, implying that all buildings at a particular location should be of the same age. The second situation, in which all housing is at saddlepoint quality, is conceptually justifiable, but empirically implausible. The stable-arm path could be profit maximizing at all locations if construction costs were high enough relative to maintenance costs, ${ }^{11}$ but casual empiricism suggests that with actual construction and maintenance technologies, construction-downgrading-demolition cycles are to be expected, at least at some locations. ${ }^{12}$ The third situation, in which a household's choice of housing quantity is invariant to its choice of housing quality (given $U$ and $Z$ ), appears to us to be the most plausible. In consequence, in both this section and the next section on comparative statics, we shall assume that $H_{q}=0$, i.e. we shall employ (AS-2).

We now complete the specification of the market-clearing model under (AS-2). For the moment we omit explicit reference to the exogenous variables $\alpha, \delta$, and $R_{A}$, and consider the endogenous determination of the variables $Y$ and $U$ that appear in the rent function, which we shall now denote as an explicit function of both income and location:

$$
P(q, U, Z)=P(q, U, Y-\alpha s(x))=P(q, U, Y, x),
$$

in terms of the old bid-rent function defined by (3) along with (5). The solutions of the supplyside submodel with this function are thus also functions of $Y$ and $U$-- and so we write $X(U, Y, x)$ (recall that $X$ denotes the vector $\left.\left(q_{0}, q_{T}, T\right)\right), \mu(U, Y, x)$, and $\Pi(U, Y, x)$ for these solutions. We define the city boundary, located at $\bar{x}(U, Y)$, by the equation

\footnotetext{
${ }^{11}$ An increase in construction costs shifts the $\phi=K_{q} / \mu$ locus upwards (see Figure 1), which reduces Area A and increases Area B, increasing the relative profitability of the stable arm.

${ }^{12}$ In ADP2, under the assumption that housing quantity is fixed, it is shown that the stable-arm path is "more likely" optimal the closer is housing to the city center.
} 
February 21, 199617:16

$$
\Pi(U, Y, \bar{x}(U, Y))=0
$$

Consequently we can compute the number of households in the city in market equilibrium, by integrating an expression analogous to (25) over $x$.

$$
N(U, Y)=\int_{0}^{\bar{x}(U, Y)} \theta(x) \frac{\mu(U, Y, x)}{H(U, Y, x)} d x,
$$

where $H(U, Y, x)$ is just the (now $q$-independent) function of the demand-side submodel, given by (6) and with partial derivatives given by (7). The first equation of the market-clearing model is thus

$$
N(U, Y)=\bar{N}
$$

for the exogenous number $\bar{N}$.

The model could be closed by writing simply

$$
Y=I
$$

for an exogenous income $I$. This would correspond to an assumption of absentee landlords for the city. More interesting is to divide the developer's profits equally among the city dwellers -- the case of internal ownership (see Pines and Sadka (1986)). Thus define

$$
\sigma(U, Y, \bar{N})=\frac{r}{\bar{N}} \int_{0}^{\bar{x}(U, Y)} \theta(x) \Pi(U, Y, x) d x
$$

The function $\sigma$ is interpreted as each household's share of the interest on the discounted value of total profits from development in the city and is non-negative by construction. Then the equation

$$
Y=I+\sigma(U, Y, \bar{N})
$$

can be adjoined to (30) to close the model.

We now examine the existence and uniqueness of equilibrium with internal ownership. 
February 21, 199617:16

The two equations which characterize any equilibrium that may exist are (30) and (33)! 13 The functions $N$ and $\sigma$ appearing in these equations are defined by (29) and (32). The various functions that appear in these definitions can be obtained as follows. One starts from the bid-rent function of the demand-side model, $P(q, U, Y, x)$ (equations (3), (4), and (27)), and uses this in the supply-side model to obtain solutions $q_{0}, q_{T}, T, \mu$, and $\Pi$, all functions of $(U, Y, x)$. The city boundary is located at radius $\bar{x}(U, Y)$ given by (28). Next, $\theta(x)$, the area of usable land per unit radial displacement at radius $x$, is given exogenously, and must satisfy

(AS-8) The integral of the function $\theta(x), \int_{0}^{x} \theta(y) d y$, tends to infinity with $x$; in other words, there is an infinite amount of land area potentially available for development. Also $\theta(x)>0$ at all locations, except that $\theta(0)=0$.

Finally $H(U, Y, x)$ is derived from the demand-side model (equations (5), (6) and (7)). Note the absence of any dependence on $q$, because of (AS-2).

The natural way to proceed would appear to be to prove that the graphs of (30) and (33) intersect (existence) only once (uniqueness) in $(U, Y)$ space. For reasons that will be explained, our method of proof of existence and uniqueness of equilibrium proceeds somewhat differently. Unless noted otherwise, the signs of partial derivatives employed in the proof are obtained from Tables 1 and 2 .

Lemma 1: $\quad \sigma_{Y}=\frac{1}{\bar{N}} \int_{0}^{\bar{x}(Y, U)} \theta(x) \frac{\mu(U, Y, x)}{H(U, Y, x)} d x=\frac{N(U, Y)}{\bar{N}}$.

Proof: $\quad$ Since $\Pi(U, Y, \bar{x})=0$ (see (28)), from (32)

$$
\sigma_{Y}=\frac{r}{\bar{N}} \int_{0}^{\bar{x}(U, Y)} \theta(x) \Pi_{Y}(U, Y, x) d x
$$

Now consider $\Pi_{Y}$. To evaluate it, we must proceed through the function $P$. We know that

\footnotetext{
${ }^{13}$ One may view (30) as characterizing equilibrium in the housing market, and (33) as an income $=$ expenditure condition.
} 
February 21, 199617:16

$P_{Y}=1 / H>0$, (see (4), (5), and (6)), and $P_{q Y}=0$ (see (5) and (10)). Consequently a shift in $Y$ gives rise to a parallel shift of the function $P$, as in the first of the comparative statics exercises in section 3 (increase in $\chi$ ). From that exercise, we know that $\Pi_{P}=\mu / r$, and consequently, $\Pi_{Y}=\mu / r H$. (Also, from Section 3 or Table 2, $\mu_{Y}>0$, but this does not provide a useful expression for the derivative.) Substitution of this expression for $\Pi_{Y}$ into (i) and use of (29) gives the stated result. $\grave{A}$

Next, a lemma concerning $\sigma_{Y Y}$.

Lemma 2: $\quad \bar{N} \sigma_{Y Y}(U, Y, \bar{N})=N_{Y}(U, Y)>0$.

Proof: $\quad$ The equality in the result is immediate from Lemma 1. Three facts demonstrate the inequality. First, we have seen that $\mu_{Y}$ is positive (see the proof of Lemma 1), and so the numerator of the integrand in the expression (29) for $N(U, Y)$ is increasing in $Y$. Second, since $H_{Y}<0$ from (5) and (7b), the denominator of the integrand in (29) is decreasing in $Y$. Third, since $\Pi_{x}$ has the opposite sign to $\Pi_{Y}$, which is positive, $\Pi_{x}<0$. But then from the definition of $\bar{x}$ in (28) it follows that $\bar{x}_{Y}>0$, so that the upper limit of integration in (29) is increasing in $Y$. This establishes what we wished to show.À

The next result we need is expressed in the following lemma:

Lemma 3: $\quad$ Under (AS-8), for fixed $U, N(U, Y)$ tends to infinity with $Y$.

Proof: The idea of the proof is the following. We show that it is always possible for given $U$ to attain a positive level of $\mu / H$ at location $x=0$ by increasing $Y$ sufficiently. Then we see that this same level of $\mu / H$ can be attained, by further increasing $Y$, at any location $x$. Since $\mu / H$ does not decrease with $Y,(\mathrm{AS}-8)$ means that $N(Y, U)$ can be made to exceed any prespecified bound by choosing $Y$ large enough.

Choose any $q$ in the interval $(0, \hat{q})$ (recall (AS-4)) and some $P>0$. Set $Y=E(U, P, q)$ 
February 21, 199617:16

for the given value of $U$. Then, by the definition (27) of the bid-rent function, $P=P(q, U, Y, 0)$. Now let us increase $Y$ keeping the other arguments of $P$ fixed at $(q, U, 0)$. We know that $P_{Y}=1 / H>0$ (from (8) and (5)) and $P_{Y Y}>0$ (from (9) and (5)), which together imply that $P$ becomes indefinitely large with $Y$. But, by part (iii) of (AS-1a), as $P$ tends to infinity, so does expenditure on housing, $P(q, U, Y, 0) H(U, Y, 0)$. Since costs remain constant in this exercise, the profit $\Pi(U, Y, 0)$ must also become infinite with $Y$, at a rate at least as fast as that at which $P$ becomes infinite. Now recall that $P_{q Y}=0$, by (10), so that we are in the presence of a parallel shift of the rent function. From (21), therefore, we conclude that, for $Y$ large enough, the optimal density $\mu$ must be positive and bounded away from zero in order that $\Pi$ tend to infinity at least as fast as $P$. Finally, since $H(U, Y, 0)$ is positive and decreasing in $Y$ (by (7b)), we see that, for large enough $Y, \mu(U, Y, 0) / H(U, Y, 0)$ is bounded away from zero.

To see that the above result holds for any $x$, it is enough to observe that by our specification of the transportation cost function $s(x)$, in particular that it is finite for any finite $x$, there is always a finite increment in $Y$ which will exactly compensate in net income $Z$ for any finite $x$. As $Y$ tends to infinity, $\bar{x}(U, Y)$ tends to infinity, and by (AS-8) so too does the land area of the city. Since population density is bounded away from zero at all settled locations, population tends to infinity. ̀̀

A fourth lemma:

Lemma 4: $\quad$ For given $Y$ large enough and for any $\bar{N}>0, \sigma(Y, U, \bar{N})$ can be made to increase indefinitely by decreasing $U$ sufficiently.

Proof: $\quad$ This result is a little more tricky than the preceding one, since we cannot use the same comparative statics exercise with $U$ as with $Y$. But it is still clear from (32) that what we need is that $\Pi\left(U, Y, x^{\prime}\right)$ be unbounded above as $U$ decreases, where $x^{\prime}$ is fixed and positive, satisfying $\alpha s\left(x^{\prime}\right)<Y$ (with the assumed properties of $s(x)$ (see (5)) this is always possible for large enough $Y$ ). The next step is to ensure that there is always a $U$ small enough that, given $Y$, 
February 21, 199617:16

construction would take place at $x^{\prime}$, that is, $\mu\left(U, Y, x^{\prime}\right)>0$. This last condition will be satisfied if the bid rent at $x^{\prime}$ for housing of quality $\hat{q}$ is high enough. To see this, recall that, from (AS-1b), $P_{q U}>0$, so that decreasing $U$ makes the slope of the bid-rent curve less steep. Thus, an increase in the rent at quality $\hat{q}$ caused by reducing $U$ entails greater increases at lower qualities. Suppose then that, if $P\left(\hat{q}, U, Y, x^{\prime}\right)>\underline{P}$, it would be profitable to construct, that is $\mu\left(U, Y, x^{\prime}\right)>0$. But by (AS-1a), for fixed $(q, Y, x)$, we can always find a $U$ such that $P(q, U, Y, x)$ takes on any positive value, however large. Thus the entire bid-rent curve, over the full range $(0, \hat{q})$, can be made to lie above any prespecified value, by choosing $U$ low enough. By arguments similar to those used in the proof of Lemma 3, we find that $\Pi\left(U, Y, x^{\prime}\right)$ can be made indefinitely large, and thus also $\sigma(U, Y, \bar{N})$ for $Y$ large enough and $\bar{N}>0 . \grave{A}$

Lemma 5: $\quad \sigma_{U}<0$.

Proof: $\quad$ Since $P_{U}<0$ for all $q$, an increase in $U$ causes the bid-rent curve to fall for all $q$, which implies that $\Pi_{U}<0$. Since $\Pi(U, Y, \bar{x})=0$ (see (28)), it then follows from (32) that $\sigma_{U}<0 . \grave{A}$

A remark is in order. The assumption (AS-1a) is clearly critical for the proof of Lemma 4. It is perhaps instructive to give an example in which equilibrium cannot exist in order to see what the force of the assumption is. Suppose that there were some minimum positive quantity of housing that each household had to consume in order to survive. This supposition is, as we remarked in Section 2, incompatible with (AS-1a). Suppose further that exogenous income $I$ is so low that, even at the city center, the minimum rent required to make construction possible is sufficiently high that a household's expenditure on housing would exceed $I$. Then evidently no equilibrium exists. As we shall see, our proof of existence requires that it be possible to raise rents arbitrarily high by decreasing utility. This is not possible in the example, but is guaranteed by (AS-1a).

Proposition 1: $\quad$ Under (AS-1) to (AS-8), when developers' profits are distributed equally 
February 21, 199617:16

among households residents in the city, equilibrium with a time-independent rent function exists and is unique. ${ }^{14}$

Proof: $\quad$ Consider Figure 4, in which $Y$ is plotted as the independent variable and $W$ as the dependent variable. First, the $45_{i}$ line $W=Y$ is drawn. The point on this line for which $Y=I$ is on that member of the family of loci

$$
W=I+\sigma(U, Y, \bar{N})
$$

for which $U=U_{0} \equiv \sup \{U \mid \mu(U, I, 0)>0\}$, because for utility level $U_{0}$ there is no construction at location $x=0$, and so none anywhere, so that $\sigma\left(U_{0}, I, N\right)=0$. At this point we also have that $\sigma_{Y}=N(U, Y) / \bar{N}=0$ (Lemma 1 ), and so locus (35) with $U=U_{0}$ is locally flat at $Y=I$. From Lemmas 2 and 3, $\sigma_{Y Y}>0$ and $\sigma_{Y}$ increases without limit, and so the locus must cross the $45_{i}$ line for some finite $Y>I$, and can do so only once, as shown in the Figure.

The conditions (30) and (33) for equilibrium will be satisfied if a value of $U$ can be found, $U^{e}$ say, such that for that value the locus (35) has a point of tangency with the $45_{i}$ line, $W=Y$. At this point of tangency, (33) is clearly satisfied. For (30), note that the tangency means that the slope of (35) must be unity. The slope is just $\sigma_{Y}(U, Y, \bar{N})$, which by Lemma 1 is $N(Y, U) / \bar{N}$, and so (30) is satisfied.

As $U$ varies for fixed $\bar{N},(35)$ generates a one-parameter family of curves in the $(Y, W)$ plane, to the northeast of $(I, I)$, as depicted in the Figure. Each of these curves has positive slope (since $\sigma_{Y}>0$ ) and is convex (since $\sigma_{Y Y}>0$ ). Further, since $\sigma_{U}<0$ (Lemma 5), for any pair of loci the one corresponding to the lower utility level must lie strictly to the left of the other. Also, since by Lemma $4 \sigma$ can be increased without limit by reducing $U$, there must exist some $\hat{U}$

\footnotetext{
${ }^{14}$ The reader may check that if we set $\mu=1$ in the model and interpret $H(U, Y, x)$ as lot size, and $\Pi(U, Y, x)$ as the profit derived from land, then the proposition goes through with minor variations in the proof. Such a modified proposition provides a proof of existence and uniqueness of equilibrium in the classical Alonso model extended to incorporate equal ownership of land by city residents.
} 
such that (35) with $U=\hat{U}$ lies entirely above the $45 i$-line. ${ }^{15}$ Finally, on account of continuity considerations and the convexity of the loci, there must exist a unique $U^{e}$ such that (35) with $U=$ $U^{e}$ is tangent to the $45 \dot{i}$-line. ${ }^{16} \grave{\mathrm{A}}$

We now derive an equation that is important for comparative static analysis, and briefly investigate existence and uniqueness for the absentee landlord case. From (4), (5), (6), (10) (which incorporates (AS-2)), and Section 3, an increase in $x$ causes a change in $P$ that can be characterized by a value of the shift parameter $\chi$ equal to $-\alpha s^{\prime}(x) / H$. From (21), we have

$$
\Pi_{x}=-\mu \alpha s^{\prime}(x) / r H
$$

Substituting (36) into (29) yields

$$
N(U, Y)=-r \int_{0}^{\bar{x}(U, Y)}\left[\frac{\theta(x)}{\alpha s^{\prime}(x)}\right] \Pi_{x} d x .
$$

Integrating (37) by parts, as in Wheaton (1974), yields

$$
N(U, Y)=r \int_{0}^{\bar{x}(U, Y)} \Omega^{\prime}(x) \Pi(U, Y, x) d x,
$$

where we have denoted $\theta(x) / \alpha s^{\prime}(x)$ by $\Omega(x)$, and used the facts that $\theta(0)=0$ and $\Pi(U, Y, \bar{x}(U, Y))=0$ (see (28)). Differentiating (38) with respect to $U$ gives

$$
N_{U}(U, Y)=r \int_{0}^{\bar{x}(U, Y)} \Omega^{\prime}(x) \Pi_{U}(U, Y, x) d x
$$

where we have again used $\Pi(U, Y, \bar{x}(U, Y))=0$.

It is very reasonable to make the following assumption:

$$
\Omega^{\prime}(x)>0 \text {, where } \Omega(x) \equiv \theta(x) / \alpha s^{\prime}(x) .
$$

\footnotetext{
${ }^{15}$ This is where (AS-1a) is crucial, since it is necessary to prove Lemma 4.

${ }^{16}$ It should now be apparent why we proved existence and uniqueness of equilibrium in $(Y, W)$ space rather than in $(Y, U)$ space. The proof used the result $\sigma_{Y Y}>0$, which cannot be portrayed in $(Y, U)$ space.
} 
February 21, 199617:16

For example, this assumption is clearly satisfied under the standard assumptions that $\theta(x)=2 \pi x$ (two-dimensional city with no gaps) and $s^{\prime \prime}(x)=0$ (linear transportation costs). We now have

Lemma 6: With (AS-1) to (AS-9), $N_{U}<0$ and $\sigma_{Y U}<0$. Further, $N(U, Y)$ and $\sigma_{Y}(U, Y, \bar{N})$ can be made indefinitely large by decreasing $U$.

Proof: $\quad$ The first statement follows immediately from (39), (AS-9), $\Pi_{U}<0$, and Lemma 1. The proof of the remainder is exactly analogous to the proofs of Lemmas 3 and 4.À

Proposition 2: $\quad$ Under (AS-1) to (AS-9), when profits go to absentee landlords, equilibrium with a time-dependent rent function exists and is unique.

Proof: The equilibrium conditions are (30) and (31), and are satisfied at a point in the $(Y, W)$ plane that lies on the vertical line $Y=I$ at which the slope of the locus (35) passing through that point is unity (see Lemma 1). That such a point exists and is unique follows from the observations that at $(I, I)$ the slope is zero, and that as one decreases $U$ and moves up the line $Y=I$ the slope increases without limit, since $\sigma_{Y U}<0$ and $\sigma_{Y}$ tends to infinity as $U$ decreases, by Lemma 6.À

\section{COMPARATIVE STATICS IN THE MARKET-CLEARING MODEL}

This section illustrates the derivation of comparative static results by treating the effects of an increase in one of the shift parameters, non-rental income $I$. An Appendix which is available upon request treats shifts in four other shift parameters, $\bar{N}, \alpha, \delta$, and $R_{A}$. All results are summarized in Table 3 at the end of this section.

Wheaton (1974) presented the first comparative static analysis of a monocentric urban area that used general functional forms. He used the simplest urban model, in which household utility depends on a composite good and land, addressed both open cities (utility fixed, population 
February 21, 199617:16

endogenous) and closed cities (population fixed, utility endogenous). Brueckner (1987) added housing and structural density to the model, thereby analyzing the comparative statics of the Muth (1969) - Mills (1967, 1972) model, and generalizing their comparative static results, which assumed particular functional forms. Household utility depended on a composite good and housing quantity. Pines and Sadka (1986) extended Wheaton's work by examining a fully closed city, in which land rents accrue to urban area residents rather than to absentee landlords, so that per capita income is endogenous. Our comparative static analysis incorporates all of these extensions. In addition, it differs from the static models cited above by treating housing quality as well as quantity, and incorporating a dynamic model in which maintenance and quality deterioration are important elements.

A change in a shift parameter affects the solution $(Y, U)$ of the market-clearing model (section 4). We already know, from the demand-side and supply-side submodels, the changes in other endogenous variables induced by changes in $Y$ and $U$, which are exogenous to these submodels. In general, as might be expected, most results are indeterminate. However, assuming (AS-2) and $\frac{d \mu}{d \gamma}<0$ for demolition-cycle paths (as described below) removes the indeterminacy of many comparative-static results of the overall model (see Table 3).

In the remainder of this section comparative static derivatives with $U$ and $Y$ held constant are designated by subscripts. Comparative static derivatives with $U$ and $Y$ allowed to vary are written in ordinary $(d / d \lambda)$ notation, even though other shift parameters, and in some cases $x$ and $q$, are being held constant. The exception is that comparative static derivatives of $P$, with $U$ and $Y$ allowed to vary in response to changes in shift parameters, are designated by partial derivatives, so as to enable us to write mixed partial derivatives of $P$ with respect to $x, q$, and a shift parameter.

Consider now an increase in $I$, with the other shift parameters $\left(\bar{N}, \alpha, \delta\right.$, and $\left.R_{A}\right)$ held constant. We first determine the effect of the increase in $I$ on the equilibrium values of $U$ and $Y$. 
February 21, 199617:16

We then consider how these changes in $U$ and $Y$ alter the rent function. And finally we examine how the perturbation of the rent function affects the developer's decision.

Lemma 7: $\quad$ Under (AS-1) to (AS-9), $d U / d I>0$ and $d Y / d I>0$.

Proof: $\quad$ Equilibrium is characterized by the pair of equations (30) and (33). Using Lemma $1,(30)$ can be rewritten as

$$
\sigma_{Y}(U, Y ; \bar{N})-1=0
$$

Total differentiation of (i) and (33) gives

$$
\begin{aligned}
& \sigma_{Y U} \frac{d U}{d I}+\sigma_{Y Y} \frac{d Y}{d I}=0 \\
& \frac{d Y}{d I}=1+\sigma_{U} \frac{d U}{d I}+\sigma_{Y} \frac{d Y}{d I}
\end{aligned}
$$

Since $\sigma_{Y}-1=0$, from (40b) $\frac{d U}{d I}=-\frac{1}{\sigma_{U}}>0$ (Lemma 5). Then from (40a), $\frac{d Y}{d I}=-\left(\frac{\sigma_{Y U}}{\sigma_{Y Y}}\right) \frac{d U}{d I}=\frac{\sigma_{Y U}}{\sigma_{Y Y} \sigma_{U}}>0($ Lemmas $2,5,6) . \grave{A}$

The next lemma characterizes the perturbation in the rent function induced by the increases in $U$ and $Y$.

Lemma 8: $\quad$ Under (AS-1) to (AS-9):

a) There exists an $x_{1} \in(0, \bar{x})$ for which $\frac{d \Pi}{d I}=0$, and a $q_{1} \in\left(q_{T}\left(x_{1}\right), q_{0}\left(x_{1}\right)\right)$ such that $\frac{\partial P}{\partial l}=0$ at $\left(x_{1}, q_{1}\right)$.

b) There exists a function $\varphi(x, q)=0$ for which

i) $\left.\frac{\partial P}{\partial I}\right|_{\varphi=0}=0,\left.\quad \frac{d x}{d q}\right|_{\varphi=0}<0, \quad \varphi\left(x_{1}, q_{1}\right)=0$

ii) Below this function in $x-q$ space, $\frac{\partial P}{\partial I}<0, \frac{\partial(\partial P / d I)}{\partial x}>0, \frac{\partial(\partial P / \partial I)}{\partial q}>0$, 
February 21, 199617:16

$$
\frac{\partial^{2}(\partial P / \partial I)}{\partial x \partial q}=0
$$

iii) Above this function, $\frac{\partial P}{\partial I}>0, \frac{\partial(\partial P / \partial I)}{\partial q}>0, \frac{\partial^{2}(\partial P / \partial I)}{\partial x \partial q}=0$, but $\frac{\partial(\partial P / d I)}{\partial x}$ is of uncertain sign.

Proof: $\quad$ a) From (38) and (30), we have

$$
r \int_{0}^{\bar{x}(U, Y)} \Omega^{\prime}(x) \Pi(U, Y, x) d x=\bar{N} .
$$

Differentiating (41) with respect to $I$ and recalling (28) gives

$$
r \int_{0}^{\bar{x}(U, Y)} \Omega^{\prime}(x) \frac{d \Pi(U, Y, x)}{d I} d x=0 .
$$

Since $\Omega^{\prime}(x)>0$ by (AS-9), (42) implies that $\Pi$ must increase for some values of $x$ and decrease for other values of $x$, or else be unchanged for all values of $x$. Since $\frac{d \Pi(\cdot)}{d I}$ is continuous in $x$, there must exist an $x_{1} \in(0, \bar{x})$ such that $\frac{d \Pi\left(U, Y, x_{1}\right)}{d I}=0$. But then, since $P(q, x ; I)$ is continuous in $q$, there must exist a $q_{1} \in\left(q_{T}\left(x_{1}\right), q_{0}\left(x_{1}\right)\right)$ such that $\frac{\partial P\left(q_{1}, x_{1} ; I\right)}{\partial I}=0$.

b) From (5), (6), and (8),

$$
\frac{\partial P(q, x ; I)}{\partial x}=-\frac{\alpha s^{\prime}(x)}{h}
$$

Consequently,

$$
\frac{\partial(\partial P(q, x ; I) / \partial x)}{\partial I}=\left(\frac{\alpha s^{\prime}(x)}{h^{2}}\right) \frac{d h(U, P, q)}{d I} .
$$

Using the equality of mixed partials on the left-hand side and expanding the right-hand side,

$$
\frac{\partial(\partial P / \partial I)}{\partial x}=\left(\frac{\alpha s^{\prime}(x)}{h^{2}}\right)\left(h_{U} \frac{d U}{d I}+h_{P} \frac{\partial P}{\partial I}\right) .
$$

From Table 1, $h_{U}>0$ and $h_{P}<0$; from Lemma $7, \frac{d U}{d I}>0$; and from above $\frac{\partial P}{\partial I}=0$ at $\left(x_{1}, q_{1}\right)$. 
February 21, 199617:16

Thus, viewing (44) as an ordinary differential equation with dependent variable $\partial P / \partial I$ and independent variable $x$, we have that

$$
\frac{\partial(\partial P / \partial I)}{\partial x}>0 \text { for }(x, q) \text { such that } \frac{\partial P}{\partial I} \leq 0
$$

Remembering that $P(q, x, I)$ can be expressed as the bid rent function $P(q, U, Z)$, and using (5), we see that

$$
\frac{\partial(\partial P(q, x ; I) / \partial I)}{\partial q}=\frac{\partial(\partial P(q, x ; I) / \partial q)}{\partial I}=P_{q U} \frac{d U}{d I}+P_{q Z} \frac{d Z}{d I}
$$

From Lemma 7 and the $P_{q \lambda}$ row of Table 1, it follows that under (AS-2)

$$
\frac{\partial(\partial P(q, x ; I) / \partial I)}{\partial q}>0 \text { for all } x, q
$$

Eqs. (45) and (47) establish that the locus of $(x, q)$ for which $\frac{d P}{d l}=0, \varphi(x, q)=0$, is negativelysloped in $x-q$ space. This establishes i).

From (47), $\frac{\partial P}{\partial l}<0$ below $\varphi(x, q)=0$ in $x-q$ space and $\frac{\partial P}{\partial I}>0$ above the locus. The former result, along with (45), implies that $\frac{\partial(\partial P / \partial I)}{\partial x}>0$ below $\varphi(x, q)=0$. Furthermore, since $P_{q x}=0$ under $(\mathrm{AS}-2)$,

$$
\frac{\partial^{2}(\partial P / \partial I)}{\partial x \partial q}=\frac{\partial P_{q x}}{\partial I}=0
$$

These results together with (47) itself establish ii). For $(x, q)$ above $\varphi(x, q)=0$ an analogous argument -- except that (45) does not apply since $\frac{\partial P}{\partial I}>0$ in this region -- establishes iii) $\grave{A}$

Among the consequences of Lemma 8 is the following corollary.

Corollary 1: Suppose that $x_{1}$ and $q_{1}$ are defined as in part a of Lemma 8. It follows directly from part $b$ of Lemma 8 that 
February 21, 199617:16

a) $\frac{\partial P}{\partial I}<0$ at any point $(q, x)$ which satisfies $q \leq q_{1}$ and $x \leq x_{1}$, with at least one of the inequalities being strict.

b) $\frac{\partial P}{\partial I}>0$ at any point $(q, x)$ which satisfies $q \geq q_{1}$ and $x \geq x_{1}$, with at least one of the inequalities being strict.

We now consider how this perturbation of the rent function affects housing demand.

Lemma 9: $\quad$ Under (AS-1) to (AS-9), $\frac{d H}{d I}>0$ for all $x$ for which $\frac{\partial P}{\partial I}<0$ for some quality level.

Proof: $\quad$ From (6)

$$
\frac{d H}{d I}=\frac{d h}{d I}=h_{U} \frac{d U}{d I}+h_{P} \frac{d P}{d I}
$$

Using (AS-2), if $\frac{d H}{d l}>0$ at $x$ for some $q$, then $\frac{d H}{d l}>0$ at $x$ for all $q$. And from Lemmas 7 and 8 ,

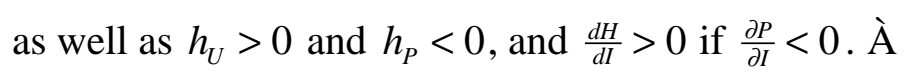

We now turn to the supply-side submodel. From (47), at each location the increase in $I$ steepens the rent function with respect to quality. This implies that if $\frac{d \Pi}{d I}=0$ at $x$, then the perturbation in the rent function corresponds to $\chi=0$ and $\gamma>0$; if $\frac{d \Pi}{d I}<0$ at $x$, then $\chi<0$ and $\gamma>0$; and if $\frac{d \Pi}{d l}>0$ at $x$, then $\chi>0$ and $\gamma>0$. Then we can apply the supply-side comparative static results given in Table 2. Thus, a crucial step is to characterize $\frac{d \Pi}{d I}$ as a function of $x$. Unfortunately, except for a couple of situations which we shall identify, the spatial pattern of $\frac{d \Pi}{d I}$ appears to be ambiguous.

Lemma 10: Under (AS-1) to (AS-9), if there is an $x_{1}$ for which $\frac{d \Pi}{d I}=0$, and for which a stablearm path is profit-maximizing, and if a stable-arm path is profit-maximizing at all locations between $x$ and $x_{1}$ :

a) If $x=x_{1}$, then at $x: \frac{d \Pi}{d I}=0, \frac{d \mu}{d I}<0, \frac{d q_{0}}{d I}>0$, and $\frac{d q_{T}}{d I}>0$ 
February 21, 199617:16

b) If $x<x_{1}$, then at $x$ : $\frac{d \Pi}{d I}<0, \frac{d \mu}{d I}<0, \frac{d q_{0}}{d I}>0$, and $\frac{d q_{T}}{d I}>0$

c) If $x>x_{1}$, then at $x: \frac{d \Pi}{d I}>0, \frac{d \mu}{d I} ?, \frac{d q_{0}}{d I}$ ?, and $\frac{d q_{T}}{d I}>0$

Proof: $\quad$ a) The change in $P(q)$ at $x$ can be characterized by a shift in the rent function $\gamma>0$. The stated result then follows from Table 2 .

b) c) Now

$$
\begin{aligned}
\frac{d(d \Pi / d I)}{d x} & =\frac{d\left(\Pi_{x}\right)}{d I} \\
& =-\frac{\alpha s^{\prime}(x)}{r}\left(\frac{1}{H} \frac{d \mu}{d I}-\frac{\mu}{H^{2}} \frac{d H}{d I}\right),
\end{aligned}
$$

where (36) is used to obtain the second equality. At any $x$ for which $\frac{d \Pi}{d l}=0, \frac{d \mu}{d l}<0$ (proof to part a)), and $\frac{d H}{d l}>0$ (Lemma 9, noting that $\frac{d P}{d l}<0$ at some quality level). Thus, at any such $x$, $\frac{d(d \Pi / d I)}{d x}>0$. This establishes that $\frac{d \Pi}{d l}<0$ for $x<x_{1}$ and $\frac{d \Pi}{d l}>0$ for $x>x_{1}$. The rest of the lemma follows from the changes in $P(q)$ implied by the sign of $\frac{d \Pi I}{d I}$, as noted above, and $\gamma>0$, along with the results from Table 2. À

The principal difficulty in establishing more general results stems from the ambiguity in the sign of $\frac{d \mu}{d \gamma}$ for the demolition cycle trajectory -- in the normal case, $\frac{d \mu}{d \gamma}<0$, but it can be positive. To obtain more general results, we assume the normal case to apply:17

${ }^{17}$ Another way to obtain more general results is to assume that structural density is fixed and constant over location. With this assumption:

- There is a unique $x, x_{1} \in(0, \bar{x})$, for which $\frac{d \Pi}{d I}=0$ and $\frac{d \Pi}{d I} \gtreqless 0$ as $x \gtreqless x_{1}$ (same line of argument used to prove Lemma 10).

- $\frac{d X}{d \lambda}=\frac{\partial X}{\partial \lambda}$ for $X=\left(q_{0}, q_{T}, T\right)$ and $\lambda=\chi, \gamma$ (the signs of $\frac{\partial X}{\partial \lambda}$ are given in Table 2).

To obtain the comparative static derivatives, proceed in the by-now-familiar way. For example, in the calculation of $\frac{d q_{0}}{d I}$ for $x<x_{1}$ : The rent gradient changes in a way characterized by $\gamma>0$ and $\chi<0$. Since $\frac{\partial q_{0}}{\partial \chi}=0$ and $\frac{\partial q_{0}}{\partial \gamma}>0$, whether the optimal path is a stable-arm path or a demolition-cycle path, $\frac{d q_{0}}{d I}>0$. 
February 21, 199617:16

(AS-10) When a demolition-cycle path is profit-maximizing, $\frac{d \mu}{d \gamma}<0$.

Lemma 11: Under (AS-1) to (AS-10):

a) There is a unique $x, x_{1} \in(0, \bar{x})$, for which $\frac{d \Pi}{d l}=0$ and $\frac{d \Pi}{d l} \gtreqless 0$ as $x \gtreqless x_{1}$.

b) If there is a stable-arm trajectory at $x$ :

$$
\begin{aligned}
& \text { - if } x \leq x_{1}, \frac{d \mu}{d I}<0, \frac{d q_{0}}{d I}>0, \frac{d q_{T}}{d I}>0 \\
& \text { - if } x>x_{1}, \frac{d \mu}{d I} ?, \frac{d q_{0}}{d I} ?, \frac{d q_{T}}{d I}>0
\end{aligned}
$$

c) If there is a demolition-cycle trajectory at $x$ :

$$
\begin{aligned}
& - \text { if } x \leq x_{1}, \frac{d \mu}{d I}<0 \text { (by (AS-10), } \frac{d q_{0}}{d I} ?, \frac{d q_{T}}{d I}>0 \\
& - \text { if } x>x_{1}, \frac{d \mu}{d I} ?, \frac{d q_{0}}{d I} ?, \frac{d q_{T}}{d I} ? \\
& \text { d) } \quad \frac{d \bar{x}}{d I}>0
\end{aligned}
$$

Proof: a) Follows from Lemma 8a and the same line of argument used to prove Lemma 10.

b) Follows from the same line of argument used to prove Lemma 10.

c) Follows from the same line of argument used to prove Lemma 10, except that the relevant comparative static derivatives drawn from Table 2 are those for a demolition-cycle path.

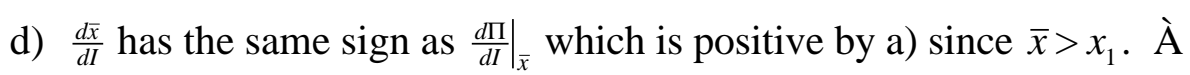

Table 3 summarizes the comparative statics of the model, with respect to changes in $I$ (derived above) and changes in $\bar{N}, \alpha, \delta$, and $R_{A}$ (derived in an Appendix, which is available on 
February 21, 199617:16

request). ${ }^{18}$ Throughout the table, (AS-1) to (AS-9) is assumed. Many results are ambiguous in general, though more may be signed if (AS-10) is employed.

\section{6. $\quad$ SUMMARY AND CONCLUDING COMMENTS}

In this paper we have investigated the properties of stationary-state general equilibrium in a monocentric city in which, on the demand side, identical households choose location, housing quality and quantity, and other goods, while on the supply side, developers choose the structural density and time path of quality of buildings. Employing a restrictive assumption on preferences, we proved existence and uniqueness of equilibrium, and determined its comparative static properties.

Most comparative static derivatives were ambiguous (most of those that are signed in Table 3 are signed on the basis of restrictive assumptions). This paucity of unambiguous comparative static results indicates that the conceptual framework is consistent with a wide range of market behavior. That the qualitative behavior of the housing market depends on the form of the technology and of tastes is to be expected.

One lesson from the paper is that, with depreciation of durable housing in a spatial model, some restrictive assumption is needed to prove the existence of a stationary-state equilibrium. Such a restrictive assumption is necessary to ensure stationary behavior, as opposed to cycles, for instance, in a stationary environment. Put alternatively, restrictive assumptions are needed to

\footnotetext{
${ }^{18}$ Pines and Sadka (1986) used a static model in which households demand land rather than housing. We could reformulate our model to allow households to construct their own housing using the composite good and land. We could then define a reduced-form utility function over the composite good, land, and location, where the location variable captures the spatial variation in the cost of producing structure on the land. (See a related discussion in footnote 10 of Brueckner (1983).) Under (AS-2), and with $\mu$ fixed and independent of location, the cost of producing structure is independent of location (since $q_{0}, q_{T}, T, m(t)$ and $\mu$ are independent of location), and so the reduced form of our model is equivalent to the model of Pines and Sadka. In fact, the comparative static results in our Table 3 concerning $U, Y$, and $\bar{x}$ are identical to those in Table 1 of Pines and Sadka concerning $u, Y$, and $\bar{x}$. Arnott, Pines and Sadka (1986) used the same model as Pines and Sadka (1986) and focused on the comparative static effects of an increase in $\alpha$.
} 
February 21, 199617:16

convert the problem, which is essentially dynamic, into one that is amenable to static equilibrium analysis. This suggests to us that the model has been developed to the point at which nonstationary-state dynamic analysis is appropriate.

Since the qualitative properties of the stationary model depend on the form of the technology and tastes, the range of qualitative behavior of any dynamic extension is likely to be very wide. Thus, quantitative analysis with careful estimation of the model's parameters will be necessary in any practical policy application. Furthermore, since a descriptively satisfactory model (with tenure choice, moving costs, multiple household groups, etc.) will be complex, simulation supplemented with partial analysis of the model's components would seem the most promising approach. Work along these lines is in progress (e.g. Anas and Arnott (1991)).

Finally, we should emphasize that our model assumes the housing market to be perfectly competitive. However, many features of housing markets (vacancy rate adjustment, search and moving costs, imperfect capital markets, etc.) are inconsistent with perfect competition. Consequently, some housing economists and many housing experts who are not economists argue that policy analysis based on competitive models of the housing market may be seriously misleading. We are agnostic, but believe that there is value to constructing sophisticated competitive models whose performance can be tested against non-competitive models as they are developed. 19

\footnotetext{
${ }^{19}$ The development of non-competitive models of the housing market is in its infancy. For a review of the
} relevant literature, see Arnott (1987). 
February 21, 199617:16

\section{REFERENCES}

Akita, T., and M. Fujita, 1982, Spatial development processes with renewal in a growing city, Environment and Planning A 14, 205-223.

Alonso, W., 1964, Location and Land Use (Harvard University Press, Cambridge, MA).

Anas, A., 1978, Dynamics of urban residential growth, Journal of Urban Economics 5, 66-87.

Anas, A. and R.J. Arnott, 1991, Dynamic housing market equilibrium with taste heterogeneity, idiosyncratic perfect foresight and stock conversions, Journal of Housing Economics 1, 2-32.

Arnott, R.J., 1980, A simple urban growth model with durable housing, Regional Science and Urban Economics 10, 53-76.

Arnott, R.J., 1987, Economic theory and housing, in: E.S. Mills, ed., Handbook of Regional and Urban Economics, Volume 2: Urban Economics, 959-988 (Elsevier, Amsterdam).

Arnott, R.J., R. Davidson, and D. Pines, 1983, Housing quality, maintenance and rehabilitation, Review of Economic Studies 50, 467-494.

Arnott, R.J., R. Davidson, and D. Pines, 1986, Spatial aspects of housing quality, density, and maintenance, Journal of Urban Economics 19, 190-217.

Arnott, R.J., D. Pines and E. Sadka, 1986, The effects of an equiproportional transport improvement in a closed monocentric city, Regional Science and Urban Economics $16,387-406$.

Braid, R.M., 1979, Theoretical Models of Urban Housing as Durable Capital, unpublished Ph.D. Dissertation, M.I.T. (1979). 
February 21, 199617:16

Braid, R.M., 1988, Uniform spatial growth with perfect foresight and durable housing, Journal of Urban Economics 23, 41-59.

Braid, R.M., 1991, Residential spatial growth with perfect foresight and multiple income groups, Journal of Urban Economics 30, 385-407.

Brueckner, J.K., 1980a, A vintage model of urban growth, Journal of Urban Economics $8,389-402$.

Brueckner, J.K., 1980b, Residential succession and land-use dynamics in a vintage model of urban housing, Regional Science and Urban Economics 10, 225-240.

Brueckner, J.K., 1981, A dynamic model of housing production, Journal of Urban Economics 10, 1-14.

Brueckner, J.K., 1983, The economics of urban yard space: An "implicit market" model for housing attributes, Journal of Urban Economics 13, 216-234.

Brueckner, J.K., 1987, The structure of urban equilibria: A unified treatment of the MuthMills model, in: E.S. Mills, ed., Handbook of Regional and Urban Economics, Volume 2: Urban Economics, 821-845 (Elsevier, Amsterdam).

Cooke, T.W., and B.W. Hamilton, 1984, Evolution of urban housing stocks: a model applied to Baltimore and Houston, Journal of Urban Economics 16, 317-338.

Debreu, G., 1959, Theory of Value: An Axiomatic Analysis of Economic Equilibrium (Yale University Press, New Haven).

Fujita, M., 1976, Spatial patterns of urban growth: optimum and market, Journal of Urban Economics 3, 209-241 (1976).

Fujita, M., 1982, Spatial patterns of residential development, Journal of Urban Economics 12, 22-52. 
February 21, 199617:16

Fujita, M. and T.E. Smith, 1987, Existence of continuous residential land-use equilibria, Regional Science and Urban Economics 17, 549-594.

Hochman, O. and D. Pines, 1982, Costs of adjustment and the spatial pattern of a growing open city, Econometrica 50, 1371-1391.

Mills, E.S., 1967, An aggregative model of resource allocation in an urban area, American Economic Review, Papers and Proceedings 57, 197-211.

Mills, E.S., 1972, Studies in the Structure of the Urban Economy (The Johns Hopkins University Press, Baltimore).

Muth, R.F., 1969, Cities and Housing (University of Chicago Press, Chicago).

Pines, D. and E. Sadka, 1986, Comparative static analysis of a fully closed city, Journal of Urban Economics 20, 1-20.

Sweeney, J.L., 1974a, Quality, commodity hierarchies, and housing markets, Econometrica 42, 147-167.

Sweeney, J.L., 1974b, A commodity hierarchy model of the rental housing market, Journal of Urban Economics 1, 288-323.

Wheaton, W.C., 1974, A comparative static analysis of urban spatial structure, Journal of Economic Theory 9, 223-237.

Wheaton, W.C., 1977, A bid-rent approach to housing demand, Journal of Urban Economics 4, 200-217.

Wheaton, W.C., 1982a, Urban residential growth under perfect foresight, Journal of Urban Economics 12, 1-21.

Wheaton, W.C., 1982b, Urban spatial development with durable but replaceable capital, Journal of Urban Economics 12, 53-67. 
February 21, 199617:16

\section{Notational Glossary}

(in alphabetical order with Roman symbols preceding Greek)

\begin{tabular}{|c|c|}
\hline$C, c$ & Hicksian composite of non-housing goods \\
\hline$E$ & expenditure function \\
\hline$F$ & cumulative distribution of building ages \\
\hline$g$ & depreciation function \\
\hline$H$ & floor space \\
\hline $\mathrm{H}$ & Hamiltonian \\
\hline$I$ & exogenous non-rental income \\
\hline$J$ & present value of rent minus maintenance cost from a building \\
\hline$K$ & construction costs \\
\hline$\ell$ & rent shift function \\
\hline$m$ & maintenance \\
\hline$N$ & population \\
\hline $\bar{N}$ & exogenous population \\
\hline$P$ & housing rent \\
\hline$q$ & quality \\
\hline$\hat{q}$ & given quality level \\
\hline$q_{0}$ & construction quality \\
\hline$q_{T}$ & terminal quality \\
\hline$Q$ & quantity of housing \\
\hline$r$ & interest rate \\
\hline$R_{A}$ & opportunity rent on land \\
\hline$s$ & transport cost \\
\hline$t$ & time \\
\hline
\end{tabular}




\begin{tabular}{|c|c|}
\hline$T$ & maximum building age \\
\hline$U, u$ & utility \\
\hline$x$ & distance from city center \\
\hline $\bar{x}$ & outer boundary of residential settlement \\
\hline$W$ & artificial variable \\
\hline$X$ & $=\left(q_{0}, q_{T}, T\right)$ \\
\hline$Y$ & total income \\
\hline$Z$ & income net of transportation costs \\
\hline$\alpha$ & transportation cost shift parameter \\
\hline$\beta$ & parameter \\
\hline$\gamma$ & housing rent shift parameter \\
\hline$\delta$ & construction cost shift parameter \\
\hline$\theta$ & residential area \\
\hline$\lambda$ & generic exogenous parameter \\
\hline$\mu$ & floor area ratio, structural density \\
\hline$\Pi$ & profit per unit area of land (differential land value) \\
\hline$\sigma$ & amortized differential land value per household \\
\hline$\tau$ & building age \\
\hline$\phi$ & co-state variable in developer's problem \\
\hline$\chi$ & housing rent shift parameter \\
\hline
\end{tabular}


February 21, 199617:16

For $H(q, U, Z)$ and $P(q, U, Z)$ :

\begin{tabular}{|c|c|c|c|c|c|}
\hline$\lambda$ & $q$ & $U$ & $Z \equiv Y-\alpha s(x)$ & $Y$ & $x$ \\
\hline$H_{\lambda}$ & $\begin{array}{c}? \\
(0 \text { with (AS- } \\
2)) \\
\end{array}$ & + & - & - & + \\
\hline$P_{\lambda}$ & + & - & + & + & - \\
\hline$P_{\mathrm{Z \lambda}}$ & $\begin{array}{c}? \\
(0 \text { with (AS- } \\
2)) \\
\end{array}$ & - & + & + & - \\
\hline$P_{q \lambda}$ & ? & $\begin{array}{c}+ \\
\text { (by (AS-1b)) }\end{array}$ & $\begin{array}{c}? \\
(0 \text { with }(A S-2))\end{array}$ & $\begin{array}{c}? \\
(0 \text { with }(\mathrm{AS}-2))\end{array}$ & $\begin{array}{c}? \\
(0 \text { with }(\mathrm{AS}-2))\end{array}$ \\
\hline
\end{tabular}

and for $h(U, P, q): h_{U}>0, h_{P}<0$, and $h_{q}$ ?

Table 1: Signs of partial derivatives from the demand-side submodel 
February 21, 199617:16

\begin{tabular}{|c|c|c|c|c|c|c|c|c|}
\hline & \multicolumn{4}{|c|}{$T$ finite } & \multicolumn{4}{|c|}{$T$ infinite } \\
\hline$\lambda$ & $\chi$ & $\gamma$ & $\delta$ & $R_{A}$ & $\chi$ & $\gamma$ & $\delta$ & $R_{A}$ \\
\hline$\frac{\partial q_{0}}{\partial \lambda}$ & 0 & + & $?$ & 0 & 0 & + & + & 0 \\
\hline$\frac{\partial q_{T}}{\partial \lambda}$ & 0 & + & + & 0 & 0 & + & 0 & 0 \\
\hline$\frac{\partial T}{\partial \lambda}$ & 0 & $?$ & $?$ & 0 & n.a. & n.a. & n.a. & n.a. \\
\hline$\frac{d q_{0}}{d \lambda}$ & $?$ & $?$ & 0 & 0 & - & + & 0 & 0 \\
\hline$\frac{d q_{T}}{d \lambda}$ & - & $(+)$ & 0 & 0 & 0 & + & 0 & 0 \\
\hline$\frac{d T}{d \lambda}$ & $?$ & $?$ & 0 & 0 & n.a. & n.a. & n.a. & n.a. \\
\hline$\frac{d \mu}{d \lambda}$ & + & $(-)$ & + & 0 & + & - & + & 0 \\
\hline$\frac{d \Pi}{d \lambda}$ & + & 0 & + & - & + & 0 & + & - \\
\hline
\end{tabular}

Notes: $1 . \frac{\partial q_{0}}{\partial \lambda}$ denotes the change in $q_{0}$ corresponding to a change in $\lambda$, holding $\mu$ fixed. $\frac{d q_{0}}{d \lambda}$ denotes the same change, but with $\mu$ changing endogenously. Etc.

2. (-) indicates that the derivative is negative under the assumption that $\frac{d \mu}{d \gamma}<0$ for a demolition-cycle path (AS-10) but is ambiguous in sign when this assumption is relaxed.

Table 2: Signs of derivatives from the supply-side submodel 
February 21, 199617:16

\begin{tabular}{|c|c|c|c|c|c|}
\hline & $I$ & $\bar{N}^{f}$ & $\alpha$ & $\delta$ & $R_{A}$ \\
\hline 1. $U$ & + & - & - & + & - \\
\hline 2. $Y$ & + & $?$ & $?$ & ? & $?$ \\
\hline 3. $\quad \bar{x}$ & $(+)$ & $?$ & $(-)$ & ? & $(-)$ \\
\hline 4. $\pi\left(x_{1}\right)^{b}$ & 0 & n.a. & (0) & 0 & 0 \\
\hline 5. $\pi(0)$ & $(-)$ & $(+)$ & $(+)$ & ? & $(+)$ \\
\hline 6. $\pi(\bar{x})$ & $(+)$ & $?$ & $(-)$ & $?$ & $(-)$ \\
\hline 7. $P\left(q_{1}, x_{1}\right)^{c}$ & 0 & n.a. & (0) & - & + \\
\hline 8. $\quad P\left(q_{3}, x_{3}\right)^{d}$ & - & n.a. & $(+)$ & - & + \\
\hline 9. $\quad P\left(q_{4}, x_{4}\right)^{e}$ & + & n.a. & $(-)$ & $?$ & $?$ \\
\hline 10. $\mu(0)$ & $(-)$ & $(+)$ & $(+)$ & $?$ & $(+)$ \\
\hline 11. $\mu(\bar{x})$ & ? & $?$ & ? & ? & ? \\
\hline 12. $H\left(q, x_{1}\right)$ & + & n.a. & $(-)$ & + & - \\
\hline 13. $H(q, 0)$ & $(+)$ & $(-)$ & $(-)$ & ? & $(-)$ \\
\hline 14. $H(q, \bar{x})$ & $?$ & $?$ & $?$ & ? & $?$ \\
\hline 15. $q_{T}(0)$ & $(+)$ & $(-)$ & $(-)$ & ? & $(-)$ \\
\hline 16. $q_{T}(\bar{x})$ & ? & ? & ? & ? & ? \\
\hline
\end{tabular}

Table 3: Comparative Statics Results

Notes:

a. Throughout the table, + indicates that the comparative static derivative of the variable in the left margin with respect to the parameter in the top margin is always positive under (AS-1) through 
February 21, 199617:16

(AS-9), whereas (+) indicates that the derivative is ambiguous but is always positive if (AS-10) is imposed as well. The symbols -, (-), 0, and (0) are defined analogously.

b. In row 4 , the entry 0 means that there is a value of $x$ between 0 and $\bar{x}$, designated $x_{1}$, for which $\Pi$ is unchanged. In each column, the value of $x_{1}$ (which generally differs among columns), depends on all of the exogenous parameters and functions of the model. Whenever there is ambiguity in rows 5 and 6 , such as in the $I$ column when (AS-10) is not imposed, it is possible that there is more than one value of $x$ for which $\Pi$ is unchanged.

c. In row 7, the entry 0 means that at $x_{1}$ (or at each value of $x_{1}$ if it is not unique), there is a unique value of $q$, designated $q_{1}$, for which $P$ is unchanged. The entry + means that at $x_{1}$, there are values of $q$, one of which is designated $q_{1}$, for which $P$ increases. Analogously for the entry -.

d. In row 8 , the entry + means that $P$ increases at any point $\left(q_{3}, x_{3}\right)$ which satisfies $q_{3} \leq q_{1}$ and $x_{3} \leq x_{1}$, with at least one of the inequalities strict. Analogously for the entry -.

e. In row 9 , the entry + means that $P$ increases at any point $\left(q_{4}, x_{4}\right)$ which satisfies $q_{4} \geq q_{1}$ and $x_{4} \geq x_{1}$, with at least one of the inequalities strict. Analogously for the entry -.

f. In the $\bar{N}$ column of the table, rows 4, 7-9, and 12 are marked n.a. (not applicable) since it is not necessarily possible to find a value of $x$, designated $x_{1}$, for which $\Pi$ is unchanged. There are always values for $x$ for which $\Pi$ increases, under (AS-1) through (AS-9), and it is possible that $\Pi$ may increase for all values of $x$. Also, there are always values of $q$ and $x$ for which $P$ increases, under (AS-1) through (AS-9).

g. The comparative static derivatives of $T, q_{0}(0)$, and $q_{0}(\bar{x})$ are not recorded since they are all ambiguous.

h. Additional comparative static derivatives can be signed if additional assumptions are made, such as (i) at all settled locations the optimal trajectory is a stable arm, or (ii) structural density is fixed. The paper contains all of the information needed to sign the derivatives under these alternative assumptions. 
February 21, 199617:16

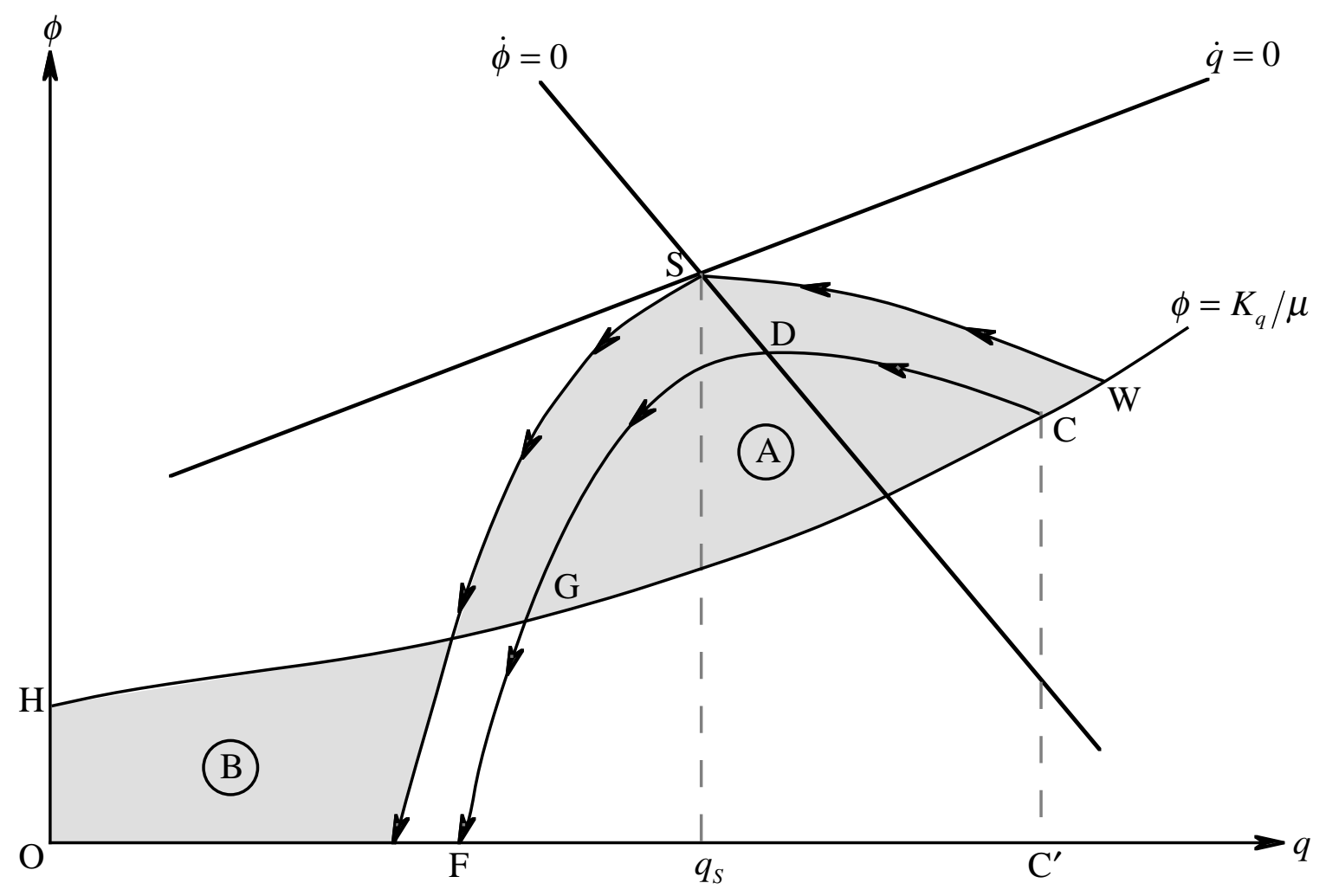

Figure 1: Phase plane for the developer's problem 
February 21, 199617:16

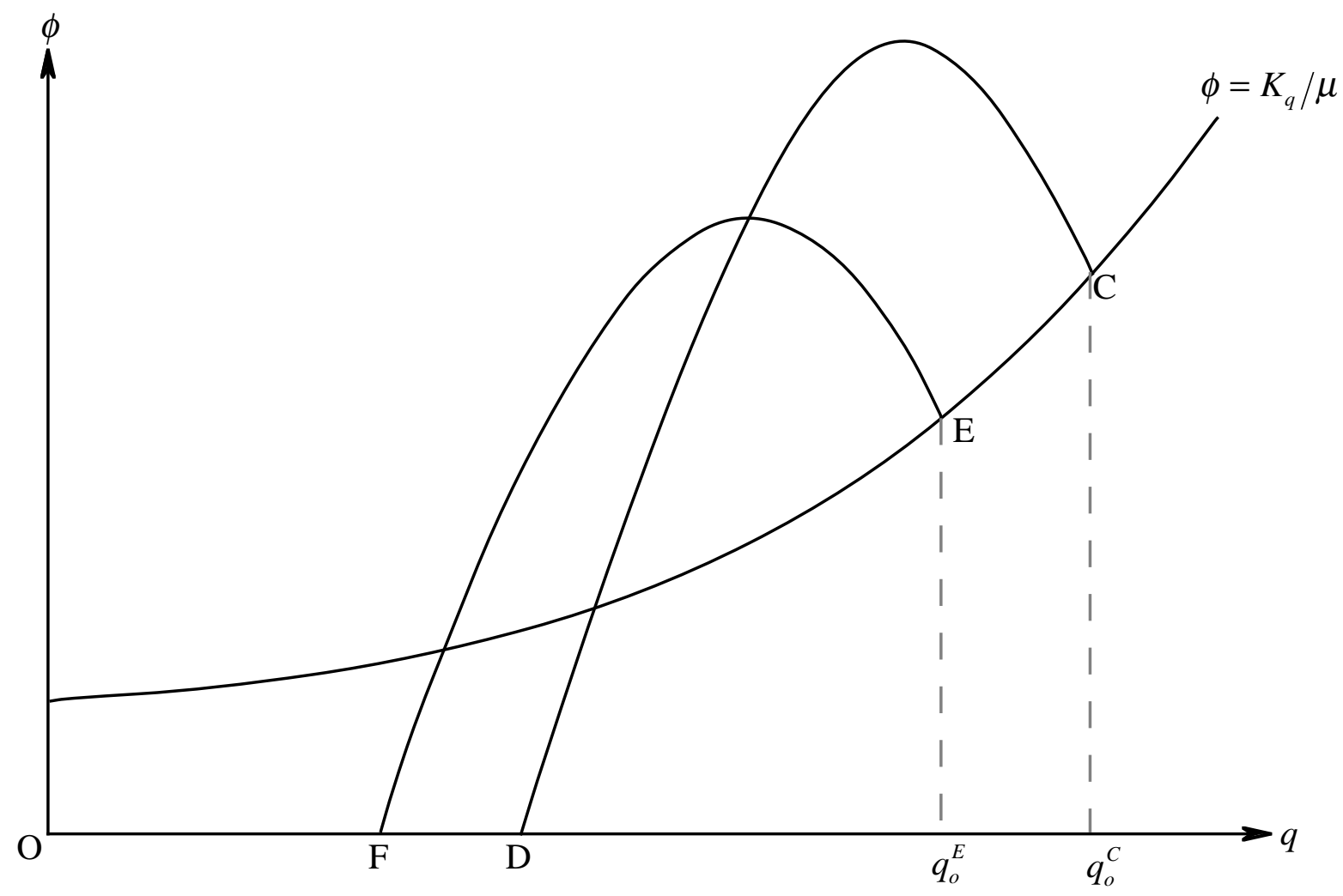

Figure 2: Demonstration that $\partial q_{O} / \partial \gamma>0, \partial q_{T} / \partial \gamma>0$ on a construction-demolition cycle 
February 21, 199617:16

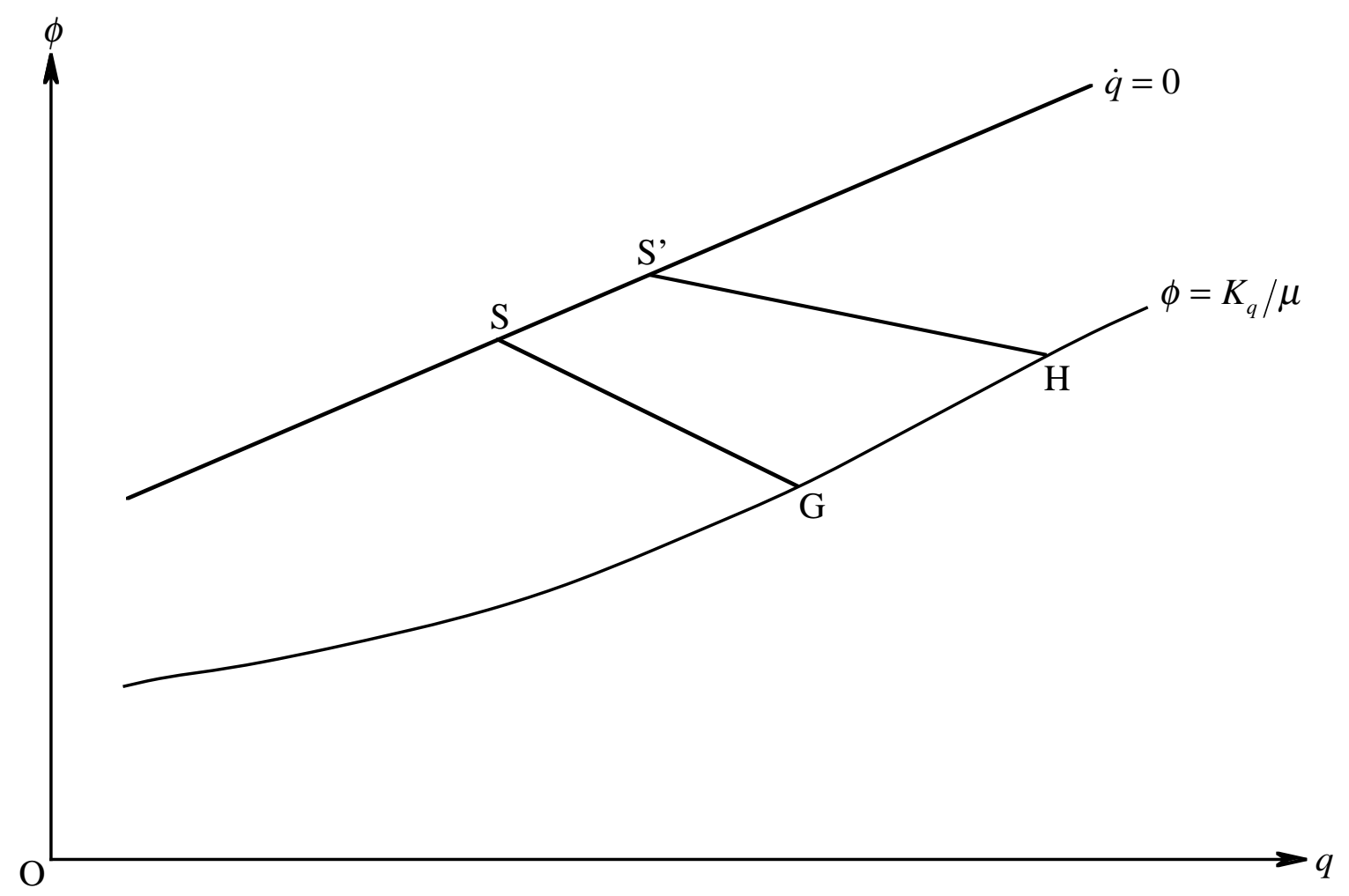

Figure 3: Demonstration that $\partial q_{O} / \partial \gamma>0$ and $\partial q_{T} / \partial \gamma>0$ on a stable arm path 
February 21, 199617:16

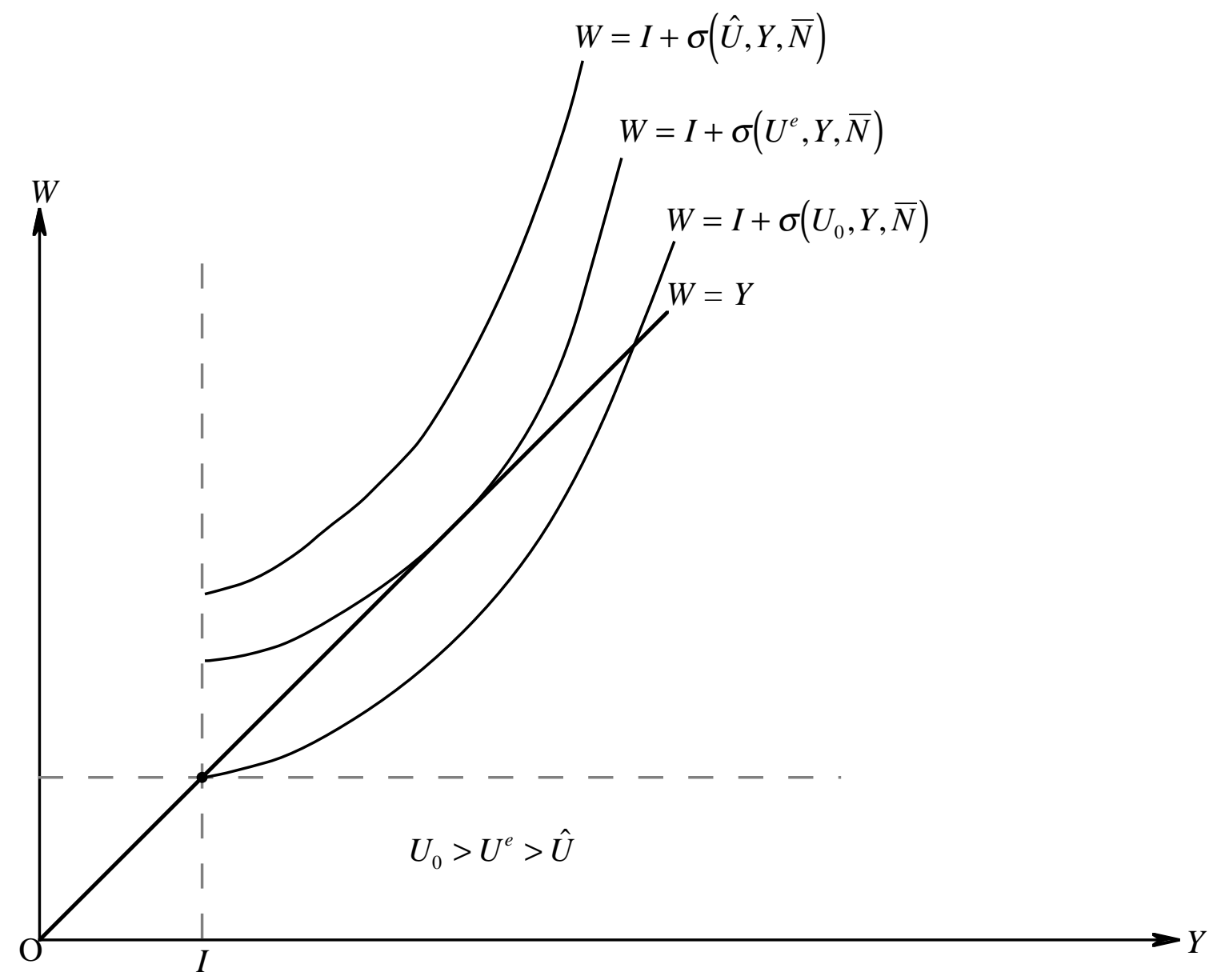

Figure 4: Proof of existence and uniqueness of equilibrium with internal ownership 
February 21, 199617:16

\section{NOT FOR PUBLICATION}

\section{$\underline{\text { APPENDIX }}$}

The comparative static analysis for $\bar{N}, \alpha, \delta$, and $R_{A}$ proceeds in much the same way as for

$I$.

1. $\bar{N}$

Lemma A1: $\quad$ Under (AS-1) to (AS-9), $\frac{d U}{d \bar{N}}<0$ and $\frac{d Y}{d \bar{N}}$ ?

Proof: $\quad$ Equilibrium is characterized by the pair of equations (30) and (33). Using Lemma 1 , (30) can be rewritten as

$$
\sigma_{Y}(U, Y, \bar{N})-1=0
$$

Total differentiation of (i) and (33) gives

$$
\begin{aligned}
& \sigma_{Y U} \frac{d U}{d \bar{N}}+\sigma_{Y Y} \frac{d Y}{d \bar{N}}+\sigma_{Y \bar{N}}=0 \\
& \frac{d Y}{d \bar{N}}=\sigma_{U} \frac{d U}{d \bar{N}}+\sigma_{Y} \frac{d Y}{d \bar{N}}+\sigma_{\bar{N}}
\end{aligned}
$$

From Lemma 1, $\sigma_{Y \bar{N}}=-\frac{\sigma_{Y}}{\bar{N}}$ which equals $-\frac{1}{\bar{N}}$ at equilibrium. From (32), $\sigma_{\bar{N}}=-\frac{\sigma}{\bar{N}}$. From (A.1b) and (i), $\frac{d U}{d \bar{N}}=-\frac{\sigma_{\bar{N}}}{\sigma_{U}}<0$ (using Lemma 5). Then from (A.1a)

$$
\begin{aligned}
\frac{d Y}{d N} & =-\frac{1}{\sigma_{Y Y}}\left(\sigma_{Y \bar{N}}-\frac{\sigma_{Y U} \sigma_{\bar{N}}}{\sigma_{U}}\right) \\
& =\frac{1}{\bar{N} \sigma_{Y Y}}\left(1-\frac{\sigma_{Y U}}{\sigma_{U}}\right)
\end{aligned}
$$

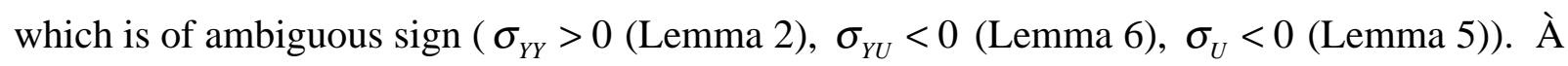

These results are intuitive. A rise in population makes land more expensive, causing 
February 21, 199617:16

differential land rents to rise. But whether differential land rents rise more or less than proportionally to the rise in population depends on the elasticities of substitution between land and capital in the production of housing, and between housing and other goods in consumption.

Lemma A2: $\quad$ Under (AS-1) to (AS-9):

a) If $\frac{d Y}{d \bar{N}}>0$, then $\frac{\partial P}{\partial \bar{N}}>0$ for all values of $x$ and $q$. Consequently, $\frac{d \Pi}{d \bar{N}}>0$ for all $x \in[0, \bar{x})$ and $\bar{x}$ increases.

b) If $\frac{d Y}{d \bar{N}}<0$, there are two possibilities. Either $\frac{d \Pi}{d \bar{N}}>0$ for all $x \in[0, \bar{x})$ and $\bar{x}$ increases. Or there is an $x_{1} \in[0, \bar{x})$ for which $\frac{d \Pi}{d \bar{N}}=0$ and a pair $\left(x_{1}, q_{1}\right)$ for which $\frac{\partial P}{\partial \bar{N}}=0$. Under the latter possibility, there exists a function $\varphi(x, q)=0$ for which

i) $\left.\quad \frac{\partial P}{\partial \bar{N}}\right|_{\varphi=0}=0,\left.\frac{d x}{d q}\right|_{\varphi=0}<0, \varphi\left(x_{1}, q_{1}\right)=0$

ii) Below the function in $x-q$ space, $\frac{\partial P}{\partial \bar{N}}>0, \frac{\partial(\partial P / \partial \bar{N})}{\partial x}<0, \frac{\partial(\partial P / \partial \bar{N})}{\partial q}<0$, $\frac{\partial^{2}(\partial P / \partial \bar{N})}{\partial x \partial q}=0$

iii) Above $\varphi(x, q)=0, \frac{\partial P}{\partial \bar{N}}<0, \frac{\partial(\partial P / \partial \bar{N})}{\partial q}<0, \frac{\partial^{2}(\partial P / \partial \bar{N})}{\partial x \partial q}=0$ but $\frac{\partial(\partial P / \partial \bar{N})}{\partial x}$ is of uncertain sign.

Proof:

a) $\frac{\partial P(q, U, Z)}{\partial \bar{N}}=P_{U} \frac{d U}{d \bar{N}}+P_{Z} \frac{d Y}{d \bar{N}}>0$ (using Lemma A1 and the results from Table 1). Then $\frac{d \Pi}{d \bar{N}}>0$ for all $x \in[0, \bar{x})$ since the developer can do at least as well as pursue the program that was optimal prior to the population increase. Since $\Pi_{x}<0$ and $\Pi(\bar{x})=0, \bar{x}$ must increase.

b) Differentiating (41) with respect to $\bar{N}$ gives

$$
r \int_{0}^{\bar{x}(U, Y)} \Omega^{\prime}(x) \frac{d \Pi(U, Y, x)}{d \bar{N}} d x=1
$$


Then either $\frac{d \Pi \bar{I}}{d \bar{N}}$ increases for all $x \in[0, \bar{x})$ and $\bar{x}$ increases. Or $\frac{d \Pi}{d \bar{N}}$ increases for some values of $x$ and decreases for others. Then by continuity, there is an $x_{1} \in[0, \bar{x})$ for which $\frac{d \Pi}{d \bar{N}}=0$ and a pair $\left(x_{1}, q_{1}\right)$ for which $\frac{\partial P}{\partial \bar{N}}=0$. Proceeding as in Lemma 8

$$
\frac{\partial(\partial P / \partial \bar{N})}{\partial x}=\frac{\alpha s^{\prime}(x)}{h^{2}}\left(h_{U} \frac{d U}{d \bar{N}}+h_{P} \frac{\partial P}{\partial \bar{N}}\right) .
$$

From Table $1, h_{U}>0$ and $h_{P}<0$; and from Lemma A1, $\frac{d U}{d \bar{N}}<0$. Thus,

$$
\frac{\partial(\partial P / \partial \bar{N})}{\partial x}<0 \text { for }(x, q) \text { such that } \frac{\partial P}{\partial \bar{N}} \geq 0
$$

Analogously to (46)

$$
\frac{\partial(\partial P / \partial \bar{N})}{\partial q}=\frac{\partial(\partial P / \partial q)}{\partial \bar{N}}=P_{q U} \frac{d U}{d \bar{N}}+P_{q Z} \frac{d Z}{d \bar{N}} .
$$

From Lemma 7 and the $P_{q \lambda}$ row of Table 1, it follows that under (AS-2)

$$
\frac{\partial(\partial P / \partial \bar{N})}{\partial q}<0 \text { for all } x, q
$$

Eqs. (A.5) and (A.7) together imply that the locus of $(x, q)$ for which $\frac{\partial P}{\partial \bar{N}}=0, \varphi(x, q)=0$, is negatively-sloped in $x$ - $q$ space. This establishes bi) of the Lemma.

From (A.7), $\frac{\partial P}{\partial \bar{N}}>0$ below $\varphi(x, q)=0$ in $x-q$ space; and $\frac{\partial P}{\partial \bar{N}}<0$ above the locus. The former result, along with (A.5), implies that $\frac{\partial(\partial P / \partial \bar{N})}{\partial \bar{x}}<0$ below $\varphi(x, q)=0$. But above the locus, $\frac{\partial(\partial P / \partial \bar{N})}{\partial \bar{x}}$ cannot be signed. Finally, since $P_{q x}=0$ by (AS-2), $\frac{\partial^{2}(\partial P / \partial \bar{N})}{\partial x \partial q}=\frac{\partial P_{q x}}{\partial \bar{N}}=0 . \grave{A}$

Lemma A3: Under (AS-1) to (AS-9), $\frac{d H}{d \bar{N}}<0$ for all $x$ for which $\frac{\partial P}{\partial \bar{N}}>0$ for some quality level.

Proof: $\quad$ Same structure as the proof to Lemma 9. À

We now turn to the supply-side submodel. There are two cases to consider. In the first, $\frac{d \Pi \bar{I}}{d \bar{N}}>0$ for all $x \in[0, \bar{x})$. Along with (A.7), this implies that the perturbation in the rent gradient 
February 21, 199617:16

can be decomposed into shifts $\chi>0$ and $\gamma<0$. In the second, $\frac{d \Pi}{d \bar{N}}$ is negative for some $x \in[0, \bar{x})$ and positive for others. Applying an analogous argument to that which was employed to prove Lemma 10, it can be shown that under (AS-10) $\frac{d \Pi}{d \bar{N}} \gtreqless 0$ as $x \lesseqgtr x_{1}$, where $x_{1}$ is the sole location at which $\frac{d \Pi \bar{I}}{d \bar{N}}=0$. Thus, the perturbation in the rent gradient entails $\gamma<0$ and $\chi \gtreqless 0$ as $x \lesseqgtr x_{1}$.

Combining the results for the two cases establishes that at $x=0, \gamma<0$ and $\chi>0$, while at $x=\bar{x}, \gamma<0$ and $\chi$ ?. Computing the corresponding signs for $\frac{d \mu}{d \bar{N}}, \frac{d q_{0}}{d \bar{N}}$, and $\frac{d q_{T}}{d \bar{N}}$ for the stable-arm and demolition-cycle cases is left to the reader.

2. $\alpha$

Lemma A4: Under (AS-1) to (AS-9), $\frac{d U}{d \alpha}<0$ and $\frac{d Y}{d \alpha}$ ?

Proof: $\quad$ Equilibrium is characterized by $\sigma_{Y}(U, Y ; \alpha)-1=0$ and $Y=I+\sigma(U, Y ; \alpha)$. Total differentiation yields

$$
\begin{aligned}
& \sigma_{Y U} \frac{d U}{d \alpha}+\sigma_{Y Y} \frac{d Y}{d \alpha}+\sigma_{Y \alpha}=0 \\
& \sigma_{U} \frac{d U}{d \alpha}+\left(\sigma_{Y}-1\right) \frac{d Y}{d \alpha}+\sigma_{\alpha}=0
\end{aligned}
$$

Thus, $\frac{d U}{d \alpha}=-\frac{\sigma_{\alpha}}{\sigma_{U}}=\operatorname{sgn}\left(\sigma_{\alpha}\right)$ since $\sigma_{U}<0$ (Lemma 5), and $\frac{d Y}{d \alpha}=-\frac{1}{\sigma_{Y Y}}\left(\sigma_{Y \alpha}+\sigma_{Y U} \frac{d U}{d \alpha}\right)$ $=-\frac{1}{\sigma_{Y Y}}\left(\sigma_{Y \alpha}-\frac{\sigma_{Y U} \sigma_{\alpha}}{\sigma_{U}}\right)$. To determine the signs of $\frac{d U}{d \alpha}$ and $\frac{d Y}{d \alpha}$, we need to obtain expressions for $\sigma_{\alpha}$ and $\sigma_{Y \alpha}$. It will prove convenient to make a transformation of variables from $x$ to $v$, where $v=\alpha s(x)$ is transport cost distance. Then

$$
x=s^{-1}\left(\frac{v}{\alpha}\right) \equiv \hat{s}\left(\frac{v}{\alpha}\right), \text { where } \hat{s}^{\prime}>0
$$

Since $\Phi(x)=\Phi\left(\hat{s}\left(\frac{v}{\alpha}\right)\right)$,

$$
\theta(x) d x=\frac{1}{\alpha} \hat{s}^{\prime} \theta\left(\hat{s}\left(\frac{v}{\alpha}\right)\right) d v .
$$


February 21, 199617:16

Also since $\Pi(U, Y, x ; \alpha)=\Pi(U, Y-\alpha s(x))=\hat{\Pi}(U, Y-v)$,

$$
\hat{\Pi}_{v}=-\Pi_{Y}
$$

And the boundary, $\bar{v}(U, Y)$, is given implicitly by

$$
\hat{\Pi}(U, Y-\bar{v}(U, Y))=R_{A}
$$

Note that $\alpha$ enters neither $\hat{\Pi}(\cdot)$ nor $\bar{v}(\cdot)$. Now, from (32),

$$
\begin{aligned}
\sigma & =\frac{\bar{r}}{N} \int_{0}^{\bar{x}(U, Y ; \alpha)} \theta(x) \Pi(U, Y, x ; \alpha) d x \\
& =\frac{\bar{r}}{N} \int_{0}^{\bar{v}(U, Y)} \frac{1}{\alpha} \hat{s}^{\prime}\left(\frac{v}{\alpha}\right) \theta\left(\hat{s}\left(\frac{v}{\alpha}\right)\right) \hat{\Pi}(U, Y-v) d v
\end{aligned}
$$

after making the transformation of variables. Integration of (A.10) by parts yields

$$
\begin{aligned}
\sigma & =\frac{r}{\bar{N}}\left\{\left(\Phi\left(\hat{s}\left(\frac{v}{\alpha}\right)\right) \hat{\Pi}\right)_{0}^{\bar{v}}-\int_{0}^{\bar{v}} \Phi\left(\hat{s}\left(\frac{v}{\alpha}\right)\right) \hat{\Pi}_{v} d v\right\} \\
& =-\frac{r}{\bar{N}} \int_{0}^{\bar{v}} \Phi\left(\hat{s}\left(\frac{v}{\alpha}\right)\right) \hat{\Pi}_{v} d v .
\end{aligned}
$$

Recalling that $\alpha$ enters neither $\hat{\Pi}$ nor $\bar{v}$, and that $-\hat{\Pi}_{v}=\Pi_{Y}=\frac{\mu}{r H}$,

$$
\begin{aligned}
\sigma_{\alpha}=- & \frac{r}{\bar{N}} \int_{0}^{\bar{v}} \hat{s}^{\prime} \frac{v}{\alpha^{2}} \theta\left(\frac{v}{\alpha}\right) \frac{\mu}{r H} d v<0 \\
\sigma_{\alpha Y}= & -\frac{r}{\bar{N}}\left(\hat{s}^{\prime} \frac{v}{\alpha^{2}} \theta\left(\frac{v}{\alpha}\right) \frac{\mu}{r H}\right)_{\bar{v}} \bar{v}_{Y} \\
& -\frac{r}{\bar{N}} \int_{0}^{\bar{v}} \hat{s}^{\prime} \frac{v}{\alpha^{2}} \theta\left(\frac{v}{\alpha}\right) \frac{d\left(\frac{\mu}{r H}\right)}{d Y} d v<0
\end{aligned}
$$

since $\bar{v}_{Y}>0$ and $\frac{d\left(\frac{\mu}{r H}\right)}{d Y}=\frac{\mu_{Y}}{r H}-\frac{\mu}{r H^{2}} H_{Y}>0$ (Tables 1 and 2). Thus, $\frac{d U}{d \alpha}<0$ and $\frac{d Y}{d \alpha}=-\frac{1}{\sigma_{Y Y}}$ $\left(\sigma_{Y \alpha}-\frac{\sigma_{Y U} \sigma_{\alpha}}{\sigma_{U}}\right)$ is of uncertain sign (using Lemmas 2,5, and 6). $\grave{A}$ 
February 21, 199617:16

This result too accords with intuition. Land effectively becomes scarcer (the amount of land within any transport cost distance falls) and whether differential land rents rise or fall depends on the relevant elasticities of substitution.

Intuitively, one expects an equiproportional increase in transport costs in a closed city to increase the profitability of housing at more central locations and to lower its profitability at more distant locations. However, we have been unable to rule out the curious possibility that the profitability of housing increase at all locations. The mechanism which generates this curiosum is as follows: Because of the fall in utility, the rent gradient as a function of quality flattens $(\gamma<0)$. In the normal demolition-cycle case, this effect by itself causes structural density to rise. But if, abnormally, it causes structural density to fall and by sufficiently much to offset the reduced housing demand caused by higher rents and transport costs, the city area has to expand to accommodate the population, requiring that the profitability of housing increase at the city's boundary. This curiosum can be circumvented by assuming (AS-10).

Lemma A5: $\quad$ Under (AS-1) to (AS-10):

a) There exists an $x_{1} \in[0, \bar{x})$ for which $\frac{d \Pi}{d \alpha}=0$, and a pair $\left(x_{1}, q_{1}\right)$ for which $\frac{d P}{d l}=0$.

b) There exists a function $\varphi(\alpha, q)=0$ for which

i) $\left.\frac{\partial P}{\partial I}\right|_{\varphi=0}=0,\left.\quad \frac{d x}{d q}\right|_{\varphi=0}<0, \quad \varphi\left(x_{1}, q_{1}\right)=0$

ii) Below this function, $\frac{\partial P}{\partial \alpha}>0, \frac{\partial(\partial P / \partial \alpha)}{\partial q}<0, \frac{\partial(\partial P / \partial \alpha)}{\partial x}<0$, and $\frac{\partial^{2}(\partial P / \partial \alpha)}{\partial x \partial q}=0$.

iii) Above the function, $\frac{\partial P}{\partial \alpha}<0, \frac{\partial(\partial P / \partial \alpha)}{\partial q}<0, \quad \frac{\partial^{2}(\partial P / \partial \alpha)}{\partial x \partial q}=0$, while $\frac{\partial(\partial P / \partial \alpha)}{\partial x}$ is of uncertain sign.

Proof: 
a) From (41),

$$
r \int_{0}^{\bar{x}(U, Y)} \Omega^{\prime}(x) \Pi(U, Y, x) d x=\bar{N} .
$$

Differentiation with respect to $\alpha$, recalling that $\Omega(x)=\frac{\theta(x)}{\alpha s^{\prime}(x)}$ and $\Omega^{\prime}(x)=\frac{1}{\alpha}\left(\frac{\theta^{\prime}}{s^{\prime}}-\frac{\theta s^{\prime \prime}}{\left(s^{\prime}\right)^{2}}\right)>0$ by (AS-9), yields

$$
r \int_{0}^{\bar{x}(U, Y)} \Omega^{\prime} \frac{d \Pi}{d \alpha} d x-\frac{\bar{N}}{\alpha}=0
$$

which implies that $\frac{d \Pi}{d \alpha}>0$ for some $x \in[0, \bar{x})$.

Next we prove that $\frac{d \Pi}{d \alpha}$ is not positive for all $x \in[0, \bar{x})$. Suppose the contrary. Then since $\frac{\partial(\partial P / \partial q)}{\partial \alpha}=P_{q U} \frac{d U}{d \alpha}<0$ (using $P_{q U}>0$ and $\frac{d U}{d \alpha}<0$ ), the increase in $\alpha$ causes a perturbation of the rent gradient characterized by $\chi>0$ and $\gamma<0$. Then by the results of Table 2 , augmented by (AS10), $\frac{d \mu}{d \alpha}>0$ for $x \in[0, \bar{x})$, whether the optimal path is a stable-arm path or a demolition-cycle path. Also, $\frac{d H}{d \alpha}<0$ for $x \in[0, \bar{x})$. To establish this, recall (49), $\frac{d H}{d \alpha}=h_{U} \frac{d U}{d \alpha}+h_{P} \frac{d P}{d \alpha}$ with $h_{U}>0, \frac{d U}{d \alpha}>0$, $h_{P}<0$, and $\frac{d P}{d \alpha}>0$ for at least some $q$ (since $\frac{d \Pi}{d \alpha}>0$ ). Thus, $\frac{d H}{d \alpha}<0$ for at least some $q$ for $x \in[0, \bar{x})$, which implies $\frac{d H}{d \alpha}<0$ for all $q$ for $x \in[0, \bar{x})$ by (AS-2). Thus, we have a situation where, relative to the initial situation, housing supply expands (housing supply at each location increases and the area of the city expands since $\frac{d \Pi}{d \alpha}>0$ elsewhere) but housing consumption contracts, which is inconsistent with equilibrium. This establishes part a) of the Lemma.

The proof to part b) follows from: $\frac{\partial P}{\partial x}=-\frac{\alpha s^{\prime}(x)}{h} ; \frac{\partial(\partial P / \partial \alpha)}{\partial x}=\frac{\partial(\partial P / \partial x)}{\partial \alpha}=-\frac{s^{\prime}(x)}{h}+\frac{\alpha s^{\prime}}{h^{2}} \frac{d h}{d \alpha}$ $=-\frac{s^{\prime}}{h}+\frac{\alpha s^{\prime}}{h^{2}}\left(h_{U} U_{\alpha}+h_{P} P_{\alpha}\right)$ which is negative if $P_{\alpha}>0$ and of ambiguous sign otherwise; $\frac{\partial(\partial P / \partial \alpha)}{\partial q}=\frac{\partial(\partial P / \partial q)}{\partial \alpha}=P_{q U} \frac{d U}{d \alpha}<0 ;$ and $\frac{\partial^{2}(\partial P / \partial x)}{\partial x \partial q}=\frac{\partial\left(\partial^{2} P / \partial x \partial q\right)}{\partial \alpha}=0 . \grave{A}$

Lemma A6: $\quad$ Under (AS-1) to (AS-10):

a) There is a unique $x, x_{1} \in[0, \bar{x})$ for which $\frac{d \Pi}{d \alpha}=0$ and $\frac{d \Pi}{d \alpha} \gtreqless 0$ as $x \lesseqgtr x_{1}$.

b) If there is a stable-arm trajectory at $x$ : 
February 21, 199617:16

$$
\begin{aligned}
& \text { - if } x \leq x_{1}, \frac{d \mu}{d \alpha}>0, \frac{d q_{0}}{d \alpha}<0, \frac{d q_{T}}{d \alpha}<0 \\
& \text { - if } x>x_{1}, \frac{d \mu}{d \alpha} ?, \frac{d q_{0}}{d \alpha} ?, \frac{d q_{T}}{d \alpha}<0
\end{aligned}
$$

c) If there is a demolition-cycle trajectory at $x$ :

$$
\begin{aligned}
& \text { - if } x \leq x_{1}, \frac{d \mu}{d \alpha}>0 \text { (by AS-10), } \frac{d q_{0}}{d \alpha} ?, \frac{d q_{T}}{d \alpha}<0 \\
& \text { - if } x>x_{1}, \frac{d \mu}{d \alpha} ?, \frac{d q_{0}}{d \alpha} ?, \frac{d q_{T}}{d \alpha} ?
\end{aligned}
$$

d) $\quad \frac{d \bar{x}}{d \alpha}<0$

Proof:

a) Now

$$
\frac{d(d \Pi / d \alpha)}{d x}=\frac{d \Pi_{x}}{d \alpha}=-\frac{s^{\prime}(x) \mu}{r H}-\frac{\alpha s^{\prime}(x)}{r}\left(\frac{1}{H} \frac{d \mu}{d \alpha}-\frac{\mu}{H^{2}} \frac{d H}{d \alpha}\right)
$$

At any $x$ for which $\frac{d \Pi}{d \alpha}=0, \frac{d \mu}{d \alpha}>0$ (since $\chi=0$ and $\gamma<0$, and using (AS-10), $\frac{d H}{d \alpha}<0$ (proof of Lemma A5). Thus, at any such $x, \frac{d(d \Pi / d \alpha)}{d x}<0$, which establishes that $\frac{d \Pi}{d \alpha}>0$ for $x<x_{1}$ and $\frac{d \Pi}{d \alpha}<$ 0 for $x>x_{1}$.

b) c) d) The rest of the lemma follows from the changes in $P(q, x)$ implied by the sign of $\frac{d \Pi}{d \alpha}$, as

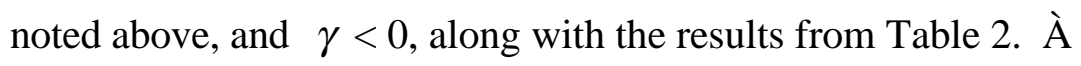

Finally, note that $\frac{\partial H}{\partial \alpha}<0$ for all $x$ for which $\frac{\partial P}{\partial \alpha}>0$ for some quality level.

\section{3. $\delta$}

Lemma A7: $\quad$ Under (AS-1) to (AS-9), $\frac{d U}{d \delta}>0$ and $\frac{d Y}{d \delta} ?$ 
Proof: $\quad$ Proceeding as in the other comparative static derivations, $\operatorname{sign}\left(\frac{d U}{d \delta}\right)=\operatorname{sign}\left(\sigma_{\delta}\right)$, while $\frac{d Y}{d \delta}=\frac{-1}{\sigma_{Y Y}}\left(\sigma_{Y \delta}-\frac{\sigma_{Y V} \sigma_{\delta}}{\sigma_{U}}\right)$.

Now, from (32)

$$
\sigma=\frac{r}{\bar{N}} \int_{0}^{\bar{x}(U, Y ; \delta)} \theta(x) \Pi(U, Y, x ; \delta) d x .
$$

Thus

$$
\sigma_{\delta}=\frac{r}{\bar{N}} \int_{0}^{\bar{x}(U, Y ; \delta)} \theta(x) \Pi_{\delta}(U, Y, x ; \delta) d x>0 \text { (Table 2). }
$$

Also, since $\Pi_{Y}=\frac{\mu}{r H}$,

$$
\sigma_{Y \delta}=\frac{1}{\bar{N}}\left\{\left.\theta(\bar{x}) \frac{\mu}{H}\right|_{\bar{x}} \bar{x}_{\delta}+\int_{0}^{\bar{x}(U, Y ; \delta)} \theta(x)\left(\frac{\mu_{\delta}}{H}-\frac{\mu}{H^{2}} H_{\delta}\right)\right\}>0 .
$$

$\bar{x}_{\delta}>0$ since with utility and income fixed, rent remains the same so that a decrease in construction costs causes profits to rise; with $U, Y$, and $x$ fixed, $H$ is unaffected by $\delta$, and $\mu_{\delta}>0$ from Table 2. Combining these results with $\sigma_{Y Y}>0$ (Lemma 2), $\sigma_{U}<0$ (Lemma 5), and $\sigma_{Y U}<0$ (Lemma 6) completes the proof. À

Again the result is intuitive. Since all "profits" accrue to residents, an improvement in the construction technology makes them better off. And whether the technological improvement, which reduces the cost of capital, causes the return to land to increase or decrease depends, $\underline{\text { inter }}$ alia, on the elasticities of substitution between land and capital in housing production and between housing and other goods in consumption.

Lemma A8: $\quad$ Under (AS-1) to (AS-9):

a) There exists an $x_{1} \in[0, \bar{x})$ for which $\frac{d \Pi}{d \delta}=0$

b) $\quad \frac{d H}{d \delta}>0$ for all $x$ for which $\frac{\partial P}{\partial \delta}<0$ for some quality level 
February 21, 199617:16

Proof: a) From (41)

$$
r \int_{0}^{\bar{x}(U, Y)} \Omega^{\prime}(x) \Pi(U, Y, x ; \delta) d x=\bar{N}
$$

Differentiation with respect to $\delta$ yields

$$
r \int_{0}^{\bar{x}(U, Y)} \Omega^{\prime}(x) \frac{d \Pi(U, Y, x ; \delta)}{d \delta} d x=0
$$

Since $\Pi(\cdot)$ is continuous, and since $\frac{d \Pi}{d \delta}$ must be positive for some $x \in[0, \bar{x})$ and negative for other $x$, there must be an $x$ for which $\frac{d \Pi}{d \delta}=0$.

b) The argument is completely analogous to that used to prove Lemma 9. À

Lemma A9: $\quad$ Under (AS-1) to (AS-9)

a) Housing rent may either fall at all settled locations or may rise at some locations and fall at others.

b) i) $\frac{\partial(\partial P / \partial \delta)}{\partial x}=\frac{\alpha s^{\prime}}{h^{s}}\left(h_{U} \frac{d U}{d \delta}+h_{P} P_{\delta}\right)$, which is positive at those locations where $P_{\delta}<0$ and of ambiguous sign otherwise.

ii) $\frac{\partial(\partial P / \partial \delta)}{\partial q}=P_{q U} \frac{d U}{d \delta}>0$, which implies that the perturbation in the rent gradient entails $\gamma>0$.

iii) $\frac{\partial^{2}(\partial P / \partial \delta)}{\partial x \partial q}=0$

Proof:

a) Housing rent cannot rise at all locations, for then profits would rise at all locations, which is inconsistent with Lemma A8a). The results in b) are easily established using earlier arguments. À

Almost all other comparative static results are ambiguous. This should not be surprising considering that we are examining a change which affects both the construction technology and the 
February 21, 199617:16

rent gradient. But it will be useful to explore further the reasons for the ambiguity.

To establish many of the comparative statics results for the changes in $I, \bar{N}$, and $\alpha-$ all demand-side changes -- we started with a location $x_{1}$ for which $\frac{d \Pi}{d \lambda}=0$. At this location, the qualitative effects of an increase in $\lambda$ on the rent function and hence (since with a demand-side change, the landlord's behavior is affected only via the rent function) on $\mu, q_{0}, q_{T}$, and $T$ (where applicable) are the same as those resulting from an increase or decrease in $\gamma$, as the case may be, which can be determined per Table 2. Furthermore, in some cases, we were able to establish, using an argument analogous to that employed in Lemma 11, that $\frac{d \Pi}{d \lambda} \gtreqless 0$ as $x_{1} \gtreqless 0$, for instance.

Then for $x<x_{1}$, for example, the qualitative effects of an increase in $\lambda$ on the rent function and hence on $\mu, q_{0}, q_{T}$, and $T$ are the same as those resulting from an increase or decrease in $\gamma$, combined with a decrease in $\chi$, which can again be determined per Table 2. Thus, in some cases we were able to ascertain the effects of the increase in $\lambda$ on $\mu, q_{0}, q_{T}$, and $T$ at all locations.

Applying the analogous procedure here -- for a supply-side change -- is problematical. First, at a location $x_{1}$ for which $\frac{d \Pi}{d \delta}=0$, the fall in construction costs must be offset by a fall in "average" rent. The qualitative effects of an increase in $\delta$ can be decomposed into the direct effect -- holding the rent function fixed -- and the indirect effects which operate via induced changes in the rent function. Thus, the qualitative effects of an increase in $\delta$ on $\mu, q_{0}, q_{T}$, and $T$ are the same as those resulting from (some average) of those resulting from an increase in $\delta$, holding the rent function fixed, an increase in $\gamma$ (Lemma A9, bii)), and a decrease in $\chi$, and are in all cases ambiguous. Second, the argument analogous to that employed in Lemma 10 is inapplicable here. That argument depended on $\mu$ and $H$ moving in opposite directions, which may well occur with a demand-side change (viz., a rise in rents typically causes construction at higher density but reduced housing consumption). But with a change in costs, structural density and housing consumption tend to move in the same direction. 
February 21, 199617:16

4. $\quad R_{A}$

Lemma A10: Under (AS-1) to (AS-9), $\frac{d U}{d R_{A}}<0$ and $\frac{d Y}{d R_{A}}$ ?

Proof: $\quad$ Proceeding as in the other comparative static derivations, $\operatorname{sign}\left(\frac{d U}{d R_{A}}\right)=\operatorname{sign} \sigma_{R_{A}}$, while $\frac{d Y}{d R_{A}}=\frac{-1}{\sigma_{Y Y}}\left(\sigma_{Y R_{A}}-\frac{\sigma_{Y U} \sigma_{R_{A}}}{\sigma_{U}}\right)$. From (32)

$$
\begin{aligned}
\sigma_{R_{A}} & =\frac{r}{\bar{N}} \int_{0}^{\bar{x}\left(U, Y ; R_{A}\right)} \theta(x) \Pi_{R_{A}}\left(U, Y, x ; R_{A}\right) d x \\
& \left.=-\frac{1}{\bar{N}} \Phi(\bar{x})<0 \text { (since } \Pi_{R_{A}}=-\frac{1}{r}\right)
\end{aligned}
$$

and

$$
\sigma_{Y R_{A}}=-\frac{1}{\bar{N}} \bar{x}_{Y} \theta(\bar{x})<0
$$

Combining these results with $\sigma_{Y Y}>0, \sigma_{U}<0$, and $\sigma_{Y U}<0$ completes the proof. $\grave{A}$

Lemma A11: Under (AS-1) to (AS-10):

a) There exists an $x_{1} \in[0, \bar{x})$ for which $\frac{d \Pi}{d R_{A}}=0$ and $\frac{d \Pi}{d R_{A}} \lesseqgtr 0$ as $x \gtreqless x_{1}$.

b) If there is a stable-arm trajectory at $x$ :

$$
\begin{aligned}
& \text { - if } x \leq x_{1}, \frac{d \mu}{d R_{A}}>0, \frac{d q_{0}}{d R_{A}}<0, \frac{d q_{T}}{d R_{A}}<0 \\
& \text { - if } x>x_{1}, \frac{d \mu}{d R_{A}} ?, \frac{d q_{0}}{d R_{A}} ?, \frac{d q_{T}}{d R_{A}}<0
\end{aligned}
$$

c) If there is a demolition-cycle trajectory at $x$ :

$$
\begin{aligned}
& \text { - if } x \leq x_{1}, \frac{d \mu}{d R_{A}}>0 \text { (by AS-10), } \frac{d q_{0}}{d R_{A}} ?, \frac{d q_{T}}{d \alpha}<0 \\
& \text { - if } x>x_{1}, \frac{d \mu}{d R_{A}} ?, \frac{d q_{0}}{d R_{A}} ?, \frac{d q_{T}}{d R_{A}} ?
\end{aligned}
$$


February 21, 199617:16

d) $\quad d \bar{x} / d R_{A}<0$

Proof:

a) Differentiation of (41) with respect to $R_{A}$ yields

$$
r \int_{0}^{\bar{x}\left(U, Y ; R_{A}\right)} \Omega^{\prime} \frac{d \Pi}{d R_{A}} d x=0
$$

which by continuity establishes that there is an $x_{1} \in[0, \bar{x})$ for which $\frac{d \Pi}{d R_{A}}=0$. Now the analog to (50) is

$$
\frac{d\left(d \Pi / d R_{A}\right)}{d x}=-\frac{\alpha s^{\prime}(x)}{r}\left(\frac{1}{H} \frac{d \mu}{d R_{A}}-\frac{\mu}{H^{2}} \frac{d H}{d R_{A}}\right) .
$$

We wish to sign this at $x_{1}$. Now, $\frac{\partial\left(\partial P / \partial R_{A}\right)}{\partial q}$ has the same sign as $\frac{d U}{d R_{A}}$, negative. Hence, $\gamma<0$. Furthermore, to offset the increase in costs, for profits to remain unchanged requires $\chi>0$. From Table 2, these changes in the rent function, along with the increase in $R_{A}$ (which has no direct effect), imply $\frac{d \mu}{d R_{A}}>0$. Also, $\frac{d H}{d R_{A}}=h_{U} \frac{d U}{d R_{A}}+h_{P} \frac{d P}{d R_{A}}<0$. Since $h_{U}>0, \frac{d U}{d R_{A}}<0, h_{P}<0$, and $\frac{d P}{d R_{A}}$ must increase at some quality levels, $\frac{d H}{d R_{A}}$ must decrease at some quality levels. But then by (AS2) $\frac{d H}{d R_{A}}$ must decrease at all quality levels. Hence, $\frac{d\left(d \Pi / d R_{A}\right)}{d x}<0$ at $x_{1}$, which by a now-familiar argument establishes the second part of a).

Parts b) and c) then follow straightforwardly. For $x<x_{1}$, the total change in $R_{A}$ can be decomposed into the direct (holding the rent function fixed) change in $R_{A}$ (which has no effect) along with induced changes in the rent function characterized by $\gamma<0$ and $\chi>0$. The combined effect on $\mu, q_{0}$, and $q_{T}$ can then be ascertained from Table 2. For $x>x_{1}$, the total change in $R_{A}$ can be decomposed into the direct change in $R_{A}$, along with the induced changes in the rent function, characterized by $\gamma<0$ and $\chi$ ? (though $>0$ for $x$ "not much greater than $x_{1}$ " (and perhaps for all $\left.x \in\left(x_{1}, \bar{x}\right)\right)$.

Part d) follows from $\left.\frac{d \Pi}{d R_{A}}\right|_{\bar{x}}<0$. ̊̀ 
February 21, 199617:16

For completeness, we record a couple of remaining results in

Lemma A12: Under (AS-1) to (AS-10)

a) $\quad \frac{d H}{d R_{A}}<0$ for $x \in\left(0, x_{1}\right)$ and may decrease for $x$ all the way up to $x_{1}$.

b) At each $x, \frac{\partial\left(\partial P / \partial R_{A}\right)}{\partial x}$ has the same sign as $\frac{d H}{d R_{A}}$.

\section{Proof:}

a) This result follows from $\frac{d H}{d R_{A}}=h_{U} \frac{d U}{d R_{A}}+h_{P} \frac{d P}{d R_{A}}$.

b) This result follows from $\frac{\partial\left(\partial P / \partial R_{A}\right)}{\partial x}=\frac{\alpha s^{\prime}(x)}{h^{2}}\left(h_{U} \frac{d U}{d R_{A}}+h_{P} \frac{d P}{d R_{A}}\right)$, which is analogous to (44). 